\title{
Dynamic Assessment of Sustainable Manufacturing Capability Based on Correlation Relationship for Industrial Cloud Robotics
}

\section{Sisi Tian}

Wuhan University of Technology

Xiaotong Xie

Wuhan University of Technology

Wenjun Xu ( $\nabla$ xuwenjun@whut.edu.cn )

Wuhan University of Technology https://orcid.org/0000-0001-5370-3437

Jiayi Liu

Wuhan University of Technology

Xiaomei Zhang

Wuhan University of Technology

\section{Research Article}

Keywords: Industrial cloud robotics, Sustainable manufacturing capability, Correlation relationship, Dynamic assessment

Posted Date: March 29th, 2021

DOI: https://doi.org/10.21203/rs.3.rs-283231/v1

License: (c) (i) This work is licensed under a Creative Commons Attribution 4.0 International License. Read Full License 


\title{
DYNAMIC ASSESSMENT OF SUSTAINABLE MANUFACTURING CAPABILITY BASED ON CORRELATION RELATIONSHIP FOR INDUSTRIAL CLOUD ROBOTICS
}

\author{
Sisi Tian ${ }^{1,2} \cdot$ Xiaotong $\mathrm{Xie}^{1,2} \cdot$ Wenjun $\mathrm{Xu}^{1,2^{*}}$.Jiayi $\mathrm{Liu}^{1,2} \cdot$ Xiaomei Zhang ${ }^{1,2}$
}

\begin{abstract}
The industrial cloud robotics (ICRs) integrates distributed industrial robot resources in various places to support complex task processing for multi-resource service requirements, and manufacturing capability assessment is the key link in determining the optimal service composition to realize the value-added of ICRs resources. However, the traditional evaluation method ignores the positive and negative cooperative effects of the manufacturing capability correlation among the robot individuals on the overall manufacturing capability of the ICRs composition. In addition, the problems of excessive resource consumption and serious environmental pollution in the manufacturing industry are becoming increasingly serious. The paper proposes a dynamic assessment method of sustainable manufacturing capability for ICRs based on the correlation relationship to solve above problems. Firstly, an extensible multi-dimensional indicator system of sustainable manufacturing capability is constructed. Then, multiple composition correlation relationships among ICRs are analyzed to establish the correlation assessment model. Furthermore, a set of dynamic evaluation methods is proposed, in which the evaluation indicators raw data is processed based on the service correlation model and the traditional network analytic network process method is improved based on the data correlation model. Finally, a case study is implemented to show the reasonability and effectiveness of the proposed method in assessment of sustainable manufacturing capability for ICRs.
\end{abstract}

Keywords: Industrial cloud robotics, Sustainable manufacturing capability, Correlation relationship, Dynamic assessment

\section{Introduction}

The use of industrial cloud robotics (ICRs) in intelligent manufacturing environments is based on the integration of cloud computing and industrial robots (IRs), which provides a new model for task execution and resource sharing in manufacturing industry compared to traditional IRs [1]. ICRs integrates distributed IRs resources in various places to provide ICRs services, and have the characteristics of convenient access, resource sharing and lower costs [2].

\footnotetext{
*Corresponding Author: Wenjun Xu

xuwenjun@whut.edu.cn

Sisi Tian

tiansisi@whut.edu.cn

Xiaotong Xie

xiexiaotong@whut.edu.cn

Jiayi Liu

jyliu@whut.edu.cn

Xiaomei Zhang

may125z@126.com

${ }^{1}$ School of Information Engineering, Wuhan University of

Technology, Wuhan 430070, China

${ }^{2}$ Hubei Key Laboratory of Broadband Wireless

Communication and Sensor Networks (Wuhan University of

Technology), Wuhan 430070, China
}

Manufacturing tasks in the ICRs platform are classified into two kinds: single resource service request task (SRSRTask) and multi-resource service request task (MRSRTask) [3]. For a SRSRTask [1], the ICRs platform searches all ICRs services that are qualified for its manufacturing task requirements and selects the optimal one to perform it. However, manufacturing tasks are rarely completed by simply invoking a single ICRs service [4]. The ICRs platform firstly decomposes the MRSRTask into several subtasks. Then, the ICRs platform finds qualified ICRs services for each subtask and pools the qualified ICRs services into a candidate ICRs set (CICRsS) [5]. A candidate ICRs service will be selected out of the CICRsS for each subtask to generate the composite ICRs service. Finally, in order to realize the valueadded of ICRs resources, the platform selects the optimal one from many ICRs compositions available with similar functions and different manufacturing capability to perform the task. How to use the disordered manufacturing data to obtain the production performance of ICRs in a timely and effective manner is an important means and a key link to provide the theoretical basis for determining the optimal ICRs composition. 
In addition, IRs are widely used in various fields such as automobile and electronics manufacturing fields due to its high efficiency, good reliability, high repeatability, being suitable for high-risk operations and other advantages [6]. However, the huge adoption of IRs also results in a large amount of energy consumption, which accounts for global warming and environmental destruction [7]. Sustainability is becoming an increasingly important requirement for manufacturing industry due to several established and emerging causes: environmental concerns, diminishing non-renewable resources, stricter legislation and inflated energy costs and so on [8]. Determining the optimal ICRs composition is one of the key factors at the operational level that influence production efficiency, quality, cost, resource consumption (energy efficiency) and waste emissions. Therefore, the research on the assessment of the sustainable manufacturing capability for ICRs has great theoretical value and practical significance.

Some collaborative relationships are formed among ICRs composition members, such as the order of ICRs to perform tasks [9]. And some business correlation relationships exist among ICRs service providers such as the vicious competition and benign competition. In addition, there are some potential feedbacks or dependencies between the manufacturing data of the evaluation indicators. These correlation relationships will have a significant impact that cannot be ignored on the overall manufacturing capability of the ICRs composition [10]. However, most of the current work is limited to the evaluation of the manufacturing capability of a single robot [11], or the overall evaluation of a robot production line [12]. The influencing factors of the overall manufacturing capability of the robot composition cannot be analyzed from the perspective of partial conversion to overall.

A dynamic assessment method for ICRs sustainable manufacturing capability based on the correlation relationship is proposed in this paper. Firstly, an extensible multi-dimensional indicator system is constructed from three aspects of production performance, energy consumption and environmental protection. According to the composition process of robots, various manufacturing capability correlation relationships among ICRs are analysed and summarized. Then, a dynamic assessment model which is based on the manufacturing correlations of the ICRs services is established. Correlation processing of the original information of evaluation indicator data is carried out based on the service correlation description model. In order to consider the dynamic interaction relationships among indicators and enhance the objectivity of the weight allocation method, an improved analytic network process (ANP) algorithm based on data correlation is proposed to obtain the weights. Moreover, the time weighting factor is introduced to adjust the importance of ICRs manufacturing data for different time periods. Finally, the final assessment results are obtained by combining the subjective evaluation method and the improved ANP algorithm. The rest of the paper is organized as follows: the related work is given in section 2 and the correlation assessment model is given in section 3. Section 4 introduces the dynamic assessment method based on correlation model. Section 5 verifies the reasonability and effectiveness of proposed method. Finally, conclusions are made in section 6 .

\section{Related Work}

\subsection{ICRs and Sustainable Manufacturing Capability}

The framework of ICRs towards sustainable manufacturing and the enabling methodologies were discussed [2]. In recent years, a knowledge evolution mechanism [13] of ICRs based on the approach of knowledge acquisition-interactive sharing-iterative updating was established. [14] proposed a cloud-based knowledge sharing mechanism for ICRs and a collaborative optimization method of service scheduling based on deep reinforcement learning. Existing research work related with ICRs primarily concentrates on the framework, energy consumption analysis, knowledge sharing and knowledge evolution..

The concept of manufacturing capability was firstly proposed by [15], and the manufacturing capability included factors such as cost, quality, delivery time and the relationship between the various elements. Some other researchers defined manufacturing capability as the combination of various capabilities such as design capability, simulation capability, production capability, management capability, and logistics capability in the whole life-cycle of manufacturing [16]. In order to realize the effective use and intelligent configuration of IRs in cloud manufacturing environment, [17] proposed a unified sustainable manufacturing capability of the IRs in terms of 
functional attributes, structural information, activities and process condition. The IRs manufacturing capability to perform the tasks with desired precision was evaluated by [18] using the circularity norm that evaluates the contortion degree of the benchmark circle to be machined. The multi-criteria approach [19] was used to quantify the manufacturing capability of a production system, and the manufacturing capability evaluation problem was modelled by analytical hierarchy process method. A decision support model which utilizes cloud model and TODIM (an acronym in Portuguese of interactive and multiple criteria decision making) method is proposed to handle robot selection problems with hesitant linguistic information [20]. These studies were mainly focused on manufacturing capability analysis and modelling methods, as well as evaluation and optimal-selection of manufacturing capability of single robot.

It is a crucial issue to realize sustainable development for the present and future generations, while sustainable manufacturing is an important part of sustainable development [21]. The sustainable production process should reduce negative environmental impact, improve the efficiency of energy and resources, minimize waste, provide operational safety, and improve personal health while improving the quality of the product and the process [22]. Waste is an important sustainability factor in manufacturing, and the cloud manufacturing was always used for waste minimisation. However, [23] proposed the concept of waste as a resource (valorisation opportunities from diversifying co-products, reuse, recycle and energy recovery), and the potential of cloud manufacturing was explored to characterise and evaluate alternative process routes for the valorisation of process manufacturing waste. In order to provide sustainable solutions to the modern industrial robotic cells, [24] proposed a cloud-based energy-efficient approach to minimise the energy consumption of robots during assembly in a cloud environment. In order to decrease the energy consumption on the premise of normal production process, the energy condition perception and big data analysis of ICRs were researched by [25], a data analysis model was built to analyse the energy consumption fluctuation characteristic of ICRs operating state and predict the trend of energy consumption associated with their operations. In this study, the sustainable manufacturing capability of ICRs are measured from four aspects: production capability, energy saving capability, environmental protection capability and recycling benefit capability.

\subsection{Manufacturing Capability Correlation and Evaluation Method}

[10] investigated three kinds of correlations in services composition when establishing a virtual enterprise, including the composable correlation, the business entity correlation and the statistical cooperate correlation, and the impact of each kind of correlation on the services composition process was studied. In order to provide users with better composite solutions, quality dependencies among services were modelled and considered during composite service selection to obtain more accurate quality estimations of service combinations [26]. A correlation-aware manufacturing cloud service description model was presented by [5] to characterize the quality dependence of an individual service on other related services, and a service correlation mapping model was proposed to get correlations among services automatically. [27] analysed the correlation between services and proposed a service composition optimization method that takes into account service correlation. [28] pointed out that service correlations exist widely in service-based distributed systems, and proposed that the correlation relationships not only includes the correlation between services, but also the correlation of user needs. In this paper, the correlation relationship within the ICRs composition is used in the evaluation of manufacturing capability to make the evaluation results closer to the actual situation.

The assessment methods can be generally classified into three categories: subjective assessment methods (such as AHP [29] and fuzzy comprehensive evaluation [30]), objective assessment methods (such as entropy-weighing method [31] and TOPSIS method [32]) and combination weighting approach. The subjective evaluation method was based on the subjective judgment of the decision makers, while the assessment results might be inconsistent due to different decision makers. The objective evaluation method often used the objective differences of the actual manufacturing data to distinguish the evaluation objects, but the preferences of decision makers were not well represented. Therefore, it is not feasible to use only one kind of assessment method to achieve the goals of making the results of assessment 
objective while reflecting the preference of decision makers. [33] combined the grey relation analysis method with the analytic hierarchy process method to comprehensively evaluate the human, robot, and human-robot collaboration capabilities during disassembly. [34] combined FAHP, VIKOR, and TOPSIS methods to comprehensively evaluate the processing risk of a certain part in the automobile manufacturing industry using FAHP-fuzzy VIKOR method. From the perspective of whether it can reflect the dynamic changes of the object to be evaluated over time, the evaluation methods can be divided into two categories: static evaluation [35] and dynamic evaluation [36]. In the manufacturing process with uncertainty and dynamics, static assessment methods are not applicable for ICRs manufacturing capability which is dynamically changed in the manufacturing process. A system dynamics method was used to study the dynamic process of sustainable development of enterprises, established a dynamic feedback mechanism between operable indicators, and simulated the dynamic evolution trend of various influencing factors of sustainable capabilities of enterprises in a continuous period [37]. An evaluation model [12] was proposed based on error feedback, and used it to dynamically modify time weights and indicator weights to achieve dynamic evaluation of robot manufacturing capability. Based on the comprehensive evaluation of subjective and objective information, this paper introduces a time factor and uses dynamic evaluation methods to grasp the changes in manufacturing capability of the ICRs over a period of time.

\section{ASSESSMENT MODEL}

\subsection{Dynamic Multi-Dimensional Indicators}

\subsubsection{Sustainable Assessment Indicator}

The evaluation indicator system of sustainable manufacturing capability of ICRs must be able to fully reflect the sustainable manufacturing capability of robots. In this paper, six first-level indicators, namely processing time, processing cost, processing quality, environmental protection, recycling benefits and energy consumption, are firstly determined. The processing time indicator is a key indicator to measure whether the ICRs composition can complete the production task on time. The processing quality indicator is the main factor that reflects the absolute competitiveness of the ICRs composition, and is also a key indicator that is closely related to the product qualification rate required by the cloud user. The processing cost indicator is an important means to improve the core competitiveness of the ICRs composition on the premise of guaranteeing the processing quality. The other three indicators reflect the sustainability of ICRs from four aspects: optimizing energy consumption, reducing environmental pollution, and improving recycling efficiency.

Furthermore, the six first-level indicators are subdivided respectively based on the analysis of the actual assembly process, and the second-level evaluation indicators of the sustainable manufacturing capability of the assembly robot are determined as shown in Fig1.

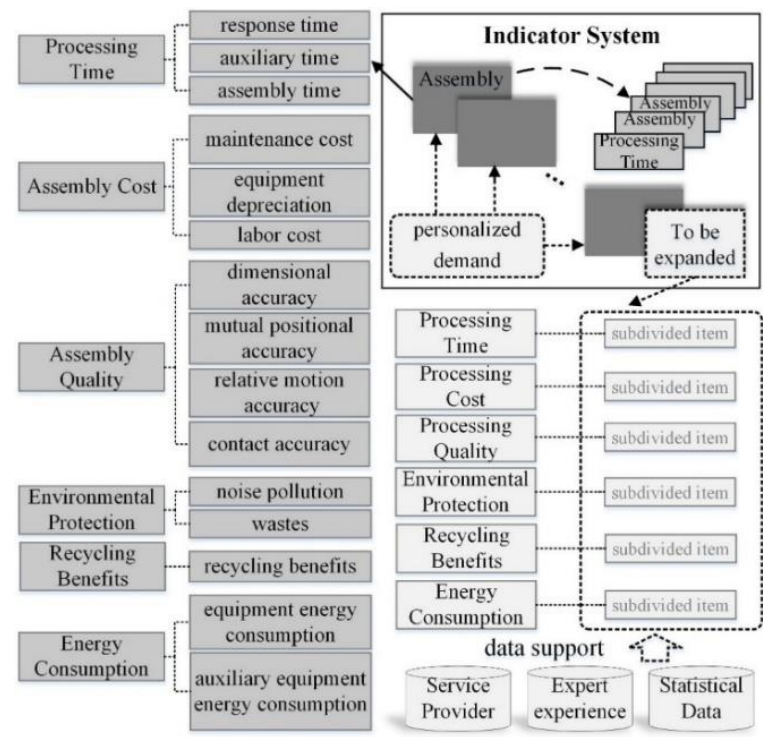

Fig1. Extensible Multi-Dimensional Indicators System 


\subsubsection{Extension Mechanism of Dynamic Indicator System}

In order to meet the needs of new types of robots in the system and the increasing personalized requirements of the indicators, a dynamic extensible evaluation indicator system is proposed as shown in Fig1.

A ICRs sustainable manufacturing capability evaluation indicator system is constructed by a two-dimensional indicator vector consisting of two elements of robot type and indicator information [38], denoted as ICRs_IndicatorSystem $=\{$ RobotType, Indicator_Info $\}$. Under these two dimensions, the indicator system is divided into several sub-indicator systems, and, each sub-indicator system consists of a six- dimensional indicator vector containing six firstlevel indicators, which is denoted as follows: Sub_IndicatorSystem $=\{$ Processing _ Time, Processing _ Cost,

Processing _Quality, Environmental_Protection,

Recycling_Benefit,Energy_Consumption

The extension mechanism of the dynamic indicator system is shown in Fig2.

\subsubsection{Quantification and Normalization of Indicators}

This paper uses the scale comparison method to quantify the two qualitative indicators of waste pollution and noise pollution, and the environmental pollution indicator quantification rules are shown in Table 1.

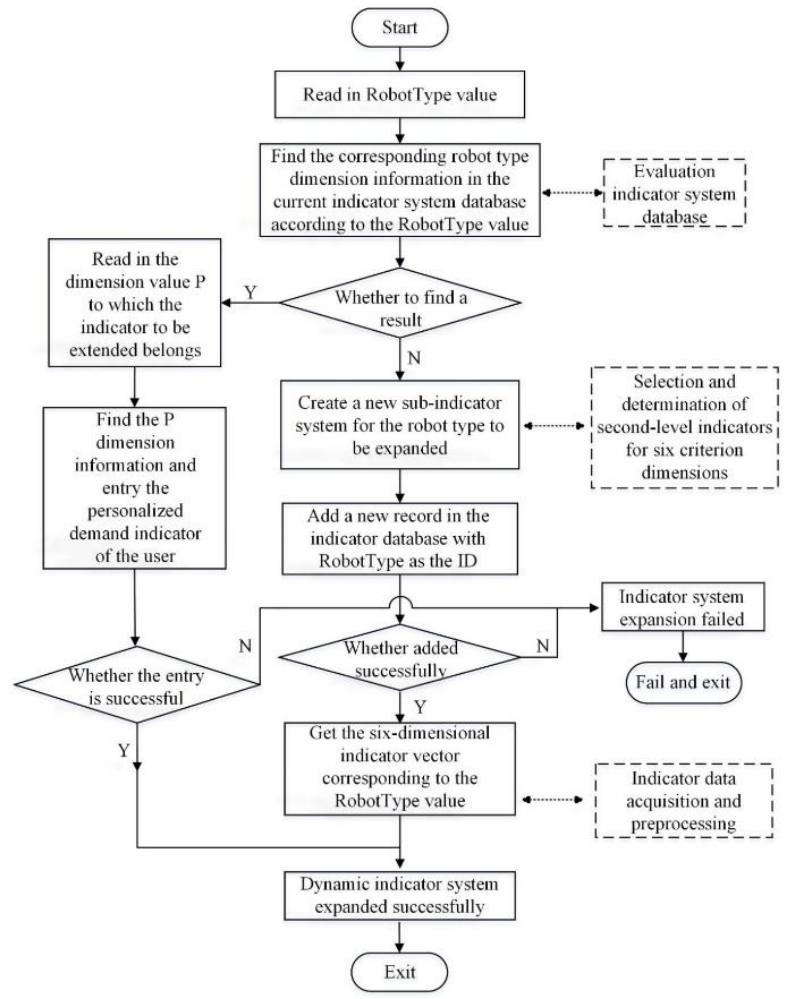

Fig2. Extension Mechanism of Dynamic Indicator System

Table 1. Environmental Pollution Indicators Quantification Rules

\begin{tabular}{|c|c|c|c|c|c|c|c|c|c|c|c|c|c|c|c|}
\hline \multirow{3}{*}{ Pollution } & \multicolumn{15}{|c|}{ Level of pollution } \\
\hline & \multicolumn{3}{|c|}{ Severe Pollution } & \multicolumn{3}{|c|}{ Moderate Pollution } & \multicolumn{3}{|c|}{ Light Pollution } & \multicolumn{3}{|c|}{ Weak Pollution } & \multicolumn{3}{|c|}{$\begin{array}{l}\text { Negligible } \\
\text { Pollution }\end{array}$} \\
\hline & $s^{+}$ & $S$ & $S^{-}$ & $m^{+}$ & $m$ & $m^{-}$ & $l^{+}$ & $l$ & $l^{-}$ & $w^{+}$ & $w$ & $w^{-}$ & $n^{+}$ & $n$ & $n$ \\
\hline Waste & 10 & 8.5 & 7.5 & 6.5 & 5.5 & 4.5 & 4.0 & 3.5 & 3.0 & 2.5 & 2.0 & 1.5 & 1.0 & 0.5 & 0 \\
\hline $\begin{array}{c}\text { Noise } \\
\text { Pollution }\end{array}$ & 10 & 9.0 & 8.0 & 7.0 & 6.0 & 5.0 & 4.0 & 3.5 & 3.0 & 2.5 & 2.0 & 1.5 & 1.0 & 0.5 & 0 \\
\hline
\end{tabular}


In order to reduce the subjective errors in the quantification of qualitative indicators as much as possible, expert credibility is introduced into the quantification process. The calculation model for the objective determination of the credibility $d_{v}$ of expert $v$ is as follows [39]:

$$
\begin{gathered}
h_{v}=1-\frac{\sqrt{\sum_{f=1}^{F}\left(y_{f}^{v}-\bar{y}_{f}\right)^{2}}}{\sqrt{\sum_{f=1}^{F}\left(y_{f}^{v}\right)^{2}}+\sqrt{\sum_{f=1}^{F}\left(\bar{y}_{f}\right)^{2}}} \\
d_{v}=\frac{h_{v}}{\sum_{v=1}^{V} h_{v}}
\end{gathered}
$$

$F$ is the total number of qualitative indicators. $y_{f}^{v}$ represents the average value of the expert group assessment of the $f$ indicator, and $V$ is the total number of the experts. The final comprehensive quantitative value of the $f$ qualitative indicator is defined as:

$$
Y_{f}=\sum_{v=1}^{V} d_{v} * y_{f}^{v}
$$

In order to eliminate the dimensional difference between a variety of quantitative indicators, the min-max normalization method is selected in this paper to achieve a unified mapping transformation of the original indicator data to the $[0,1]$ range. The min-max normalization on the sequence $x_{1}, x_{2}, \ldots, x_{n}$ is formulated as follows:

$$
y_{j}=\frac{x_{j}-\min _{1 \leq i \leq n}\left\{x_{i}\right\}}{\max _{1 \leq i \leq n}\left\{x_{i}\right\}-\min _{1 \leq i \leq n}\left\{x_{i}\right\}}
$$

Some indicators are the forward indicators, such as processing quality and recycling benefits, the larger the value of the indicator, the more prominent its contribution to the sustainable manufacturing capability of ICRs. And some other indicators are the reverse indicators, such as processing time, processing cost, environmental pollution, and energy consumption, the smaller the value of the indicator, the more it meets the expectations of users and the requirements of sustainable development. Two different min-max transformation models are adopted in this paper to standardize the indicators according to the different polarity of the indicators to make all indicators comparable. The forward indicators are transformed as follows:

$$
g_{i j}=\frac{o_{i j}-\min \left\{o_{i j}\right\}}{\max \left\{o_{i j}\right\}-\min \left\{o_{i j}\right\}}
$$

The reverse indicators are transformed as follows:

$$
g_{i j}=\frac{\max \left\{o_{i j}\right\}-o_{i j}}{\max \left\{o_{i j}\right\}-\min \left\{o_{i j}\right\}}
$$

$o_{i j}$ represents the raw data of the $j$ evaluation indicator at the $i$ moment, and $\min \left\{o_{i j}\right\}$ represents the minimum value of the $j$ indicator among all the schemes to be evaluated.

In addition, the raw sample data of processing quality types, such as dimensional accuracy, mutual position accuracy, relative motion accuracy and contact accuracy in the assembly process, is a numerical interval $\left[y_{l}, y_{u}\right]$ used to describe the maximum error of the measurement accuracy, and cannot be directly substituted into the above normalized model for calculation. The accuracy type indicator is transformed as follows:

$$
y^{f}=y_{u}
$$

The absolute values of $y_{l}$ and $y_{u}$ are usually equal, $y_{u}$ is directly selected as the final indicator value $y^{f}$.

The evaluation indicator data are processed based on the method above, and the normalized indicator values of some indicators are shown in Table 2 (EC: Equipment energy Consumption, DA: Dimensional Accuracy).

Table 2. The Normalized Indicator Values of Some Indicators

\begin{tabular}{cccccccccccc} 
EC data & 15 & 15.6 & 16 & 17 & 17.3 & 17.6 & 18.7 & 19 & 20 & 20.5 \\
$\begin{array}{c}\text { Normalize } \\
\text { d data }\end{array}$ & 1.00 & 0.93 & 0.88 & 0.75 & 0.71 & 0.68 & 0.54 & 0.50 & 0.38 & 0.31 \\
\hline DA data & \pm 0.10 & \pm 0.20 & \pm 0.25 & \pm 0.30 & \pm 0.40 & \pm 0.50 & \pm 0.55 & \pm 0.60 & \pm 0.75 & \pm 0.85 \\
$\begin{array}{c}\text { Normalize } \\
\text { d data }\end{array}$ & 0.94 & 0.83 & 0.78 & 0.72 & 0.61 & 0.50 & 0.44 & 0.39 & 0.22 & 0.11 \\
\hline
\end{tabular}




\subsection{Correlation Assessment Model}

\subsubsection{Correlation Relationships}

There will be a variety of manufacturing capability correlation relationships that cannot be ignored. When two ICRs services are composited by different execution sequence structures such as parallel structure and series structure, the contribution of each robot to the total execution time of the ICRs composition is different. if there is a business competition or cooperation relationship between two ICRs service providers, or a related relationship between two robots from the same service provider, such as a shared server, the overall manufacturing capability of the ICRs composition may be inhibited or enhanced. In the actual processing process, there may be potential manufacturing data correlations between two ICRs services or within an ICRs service, resulting in the evaluation indicators not being independent of each other. In summary, the process of transforming the manufacturing capability of robot individuals into the overall manufacturing capability of an ICRs composition is usually affected by the following three types of manufacturing capability correlation relationships: the business entity correlation relationships, the composite structure correlation relationships, and the data correlation relationships, as shown in Fig3. The first two are defined as service correlation relationships.

\subsubsection{Correlation Assessment Model}

In order to make the evaluation of ICRs composition manufacturing capability more in line with objective reality, a correlation assessment model based on the above three kinds of manufacturing capability correlation relationships is proposed in this section.

The production performance of the robot equipment and the correlation among the robot individuals of the ICRs are all changing dynamically over time, the dynamic evaluation method is used to establish the mathematical model of sustainable manufacturing capability evaluation. Firstly, the robot is evaluated statically at various time periods to obtain a set of static evaluation values based on the time series $m c_{T 1}, m c_{T 2}, m c_{T 3}, \ldots, m c_{T n}$. Then, the weight coefficients of each evaluation result at different periods are allocated $w_{T 1}, w_{T 2}, w_{T 3}, \ldots, w_{T n}$ $\left.\sum_{i=1}^{n} w_{T i}=1\right)$. Finally, the dynamic evaluation results are aggregated as $M C=\sum_{i=1}^{n} m c_{T i} w_{T i}$.

The historical manufacturing task data for $n$ time periods of the ICRs composition to be evaluated is selected as the basis for the evaluation data. $k$ ICRs compositions are denoted as $C_{1}, C_{2}, C_{3}, \ldots, C_{k}$. Each ICRs composition $C_{k}$ has $m k$ evaluation indicators $x_{k}^{1}, x_{k}^{2}, x_{k}^{3}, \ldots, x_{k}^{m k}$.

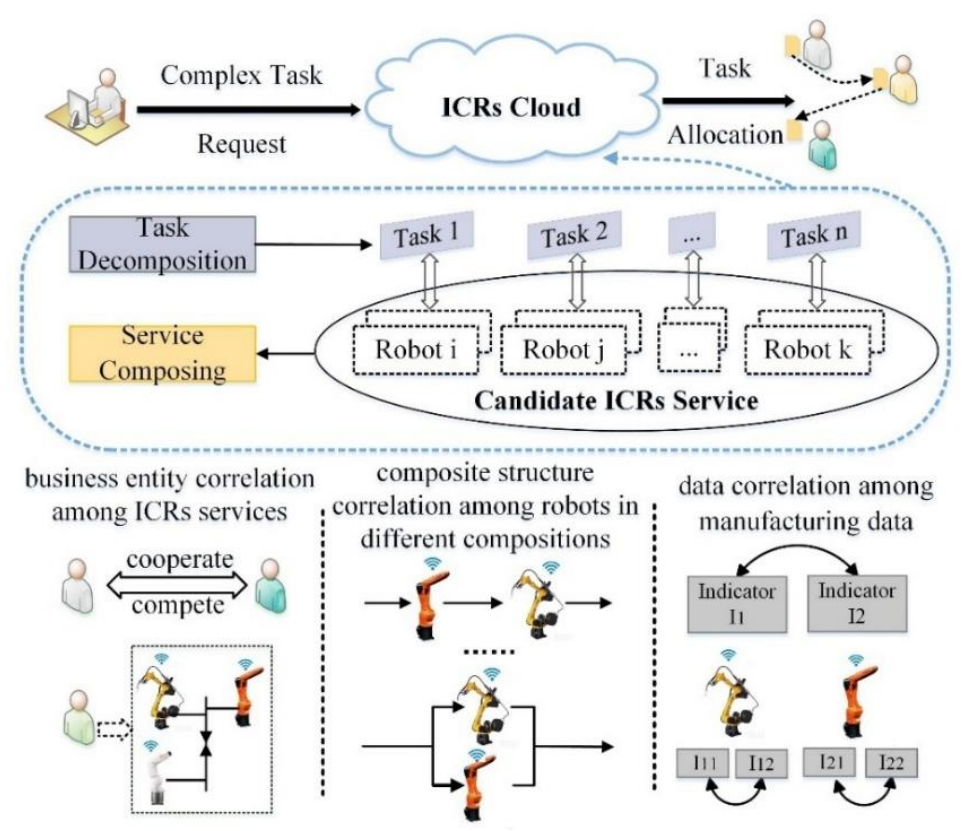

Fig3. Manufacturing Capability Correlation Relationships in ICRs Composition 


\begin{tabular}{ccccc}
\hline ICRs & $T_{1}$ & \multicolumn{2}{c}{ Indicator data of each time period } & $T_{\mathrm{n}}$ \\
composition & $x_{1}{ }^{1}\left(T_{1}\right) \ldots x_{1}{ }^{m 1}\left(T_{1}\right)$ & $x_{1}{ }^{1}\left(T_{2}\right) \ldots x_{1}{ }^{m 1}\left(T_{2}\right)$ & & $x_{1}{ }^{1}\left(T_{n}\right) \ldots x_{1}{ }^{m 1}\left(T_{n}\right)$ \\
$C_{1}$ & $x_{2}{ }^{1}\left(T_{1}\right) \ldots x_{2}{ }^{m 2}\left(T_{1}\right)$ & $x_{2}{ }^{1}\left(T_{2}\right) \ldots x_{2}{ }^{m 2}\left(T_{2}\right)$ & & $x_{2}{ }^{1}\left(T_{n}\right) \ldots x_{2}{ }^{m 2}\left(T_{n}\right)$ \\
$C_{2}$ & $\ldots$ & $\ldots$ & $\ldots$ & $\ldots$ \\
$\ldots$ & $x_{k}{ }^{1}\left(T_{1}\right) \ldots x_{k}{ }^{m k}\left(T_{1}\right)$ & $x_{k}{ }^{1}\left(T_{2}\right) \ldots x_{k}{ }^{m k}\left(T_{2}\right)$ & & $x_{k}{ }^{1}\left(T_{n}\right) \ldots x_{k}{ }^{m k}\left(T_{n}\right)$ \\
$C_{\mathrm{k}}$ &
\end{tabular}

Table 4. Data Structure of $T_{n}$

\begin{tabular}{cccc}
\hline$t_{1}$ & $t_{2}$ & $\cdots$ & $t_{l}$ \\
\hline$x_{k}{ }^{1}\left(t_{1}\right) x_{k}{ }^{2}\left(t_{1}\right) \ldots x_{k}{ }^{m k}\left(t_{1}\right)$ & $x_{k}^{1}\left(t_{2}\right) x_{k}^{2}\left(t_{2}\right) \ldots x_{k}^{m k}\left(t_{2}\right)$ & $\ldots$ & $x_{k}^{1}\left(t_{l}\right) x_{k}^{2}\left(t_{l}\right) \ldots x_{k}{ }^{m k}\left(t_{l}\right)$ \\
\hline
\end{tabular}

The indicator data information of $m k$ indicators under the time period $T_{n}$ is denoted as $x_{k}^{1}\left(T_{n}\right), x_{k}^{2}\left(T_{n}\right), x_{k}^{3}\left(T_{n}\right), \ldots, x_{k}^{m k}\left(T_{n}\right)$. Then, the time series data table of historical manufacturing data generated by $k$ robots in $n$ periods $T_{1}, T_{2}, T_{3}, \ldots, T_{n}$ is shown in Table 3 .

Time period $T_{n}$ is a whole period of continuous time composed of $l$ consecutive time nodes $t_{1}, t_{2}, t_{3}, \ldots, t_{l}$. The internal data structure of $x_{k}^{1}\left(T_{n}\right), x_{k}^{2}\left(T_{n}\right), x_{k}^{3}\left(T_{n}\right), \ldots, x_{k}^{m k}\left(T_{n}\right)$ is shown in Table 4. $x_{k}^{m k}\left(t_{l}\right)$ is the value of the $m k$ evaluation indicator of the robot composition $C_{k}$ after completing the processing task at time $t_{l}$.

Based on time series data, the static evaluation of the sustainable manufacturing capability of the ICRs composition $C_{r}$ in the time period $T_{j}$ is performed, and the evaluation function is formulated in Eq. (9).

$$
\begin{aligned}
& m c^{C_{r}}{ }_{T j}=\sum_{i=1}^{m r} w_{i} x_{r}^{i}\left(t_{\text {avg }}^{T_{j}}\right), \\
& i=1,2, \ldots, m r ; r=1,2, \ldots, k ; j=1,2, \ldots, n
\end{aligned}
$$

where $w_{1}, w_{2}, \ldots, w_{i}$ is the weight of each indicator, and satisfies the following conditions:

$$
\sum_{i=1}^{n} w_{i}=1,0 \leq w_{i} \leq 1
$$

Each period contains multiple historical manufacturing task data, the indicator data at a certain time in the period may not be broadly representative of the whole period due to its contingency. In Eq. (9), the average value $x\left(t_{\text {avg }}^{T_{j}}\right)$ of the manufacturing data at all time nodes within $T_{j}$ is selected as the evaluation indicator value of this period.

The static evaluation value $m c^{C_{r}}{ }_{T 1}, m c^{C_{r}}{ }_{T 2}, m c^{C_{r}}{ }_{T 3}, \ldots, m c^{C_{r}}{ }_{T n}$ of the sustainable manufacturing capability of $C_{r}$ under $n$ time periods is obtained, combining the time weight vector $v=\left(v_{1}, v_{2}, v_{3}, \ldots, v_{n}\right)$ $\left.\sum_{j=1}^{n} v_{j}=1,0 \leq v_{j} \leq 1\right)$ corresponding to $n$ time periods. The dynamic comprehensive evaluation of the sustainable manufacturing capability of the ICRs composition $C_{r}$ is calculated as follows:

$$
M C^{C_{r}}=\sum_{j=1}^{n} m c_{T_{j}}^{C_{r}} * v_{j}, j=1,2, \ldots, n, r=1,2, \ldots, k
$$

According to the above evaluation process and dynamic evaluation ideas, the correlation assessment model is built as shown in Fig4. 


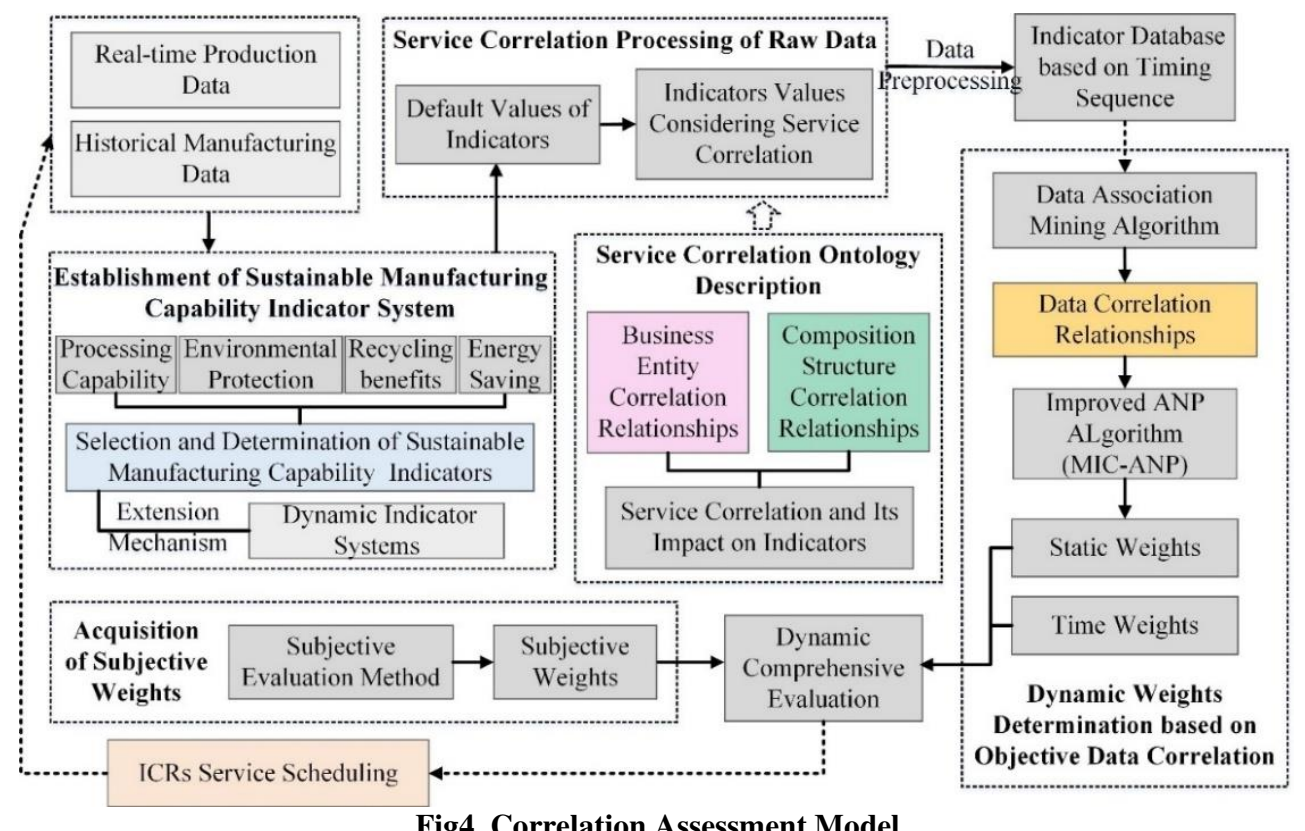

Firstly, from the four aspects of processing capability, environmental protection capability, recycling benefits capability and energy saving capability, the indicators of sustainable manufacturing capability are selected and determined. A set of multi-dimensional dynamic indicator system is constructed based on the indicator system extension mechanism. The business entity correlation relationship, the composite structure correlation relationship and their related impact on the overall manufacturing capability of the ICRs composition are analyzed. A service correlation ontology description model is established, which is used to complete the service correlation processing of the indicator raw data. In addition, the proper dynamic evaluation method is used to calculate the weight coefficient of each evaluation indicator. Firstly, the data correlation mining algorithm is used to construct the data correlation model. And the traditional ANP is improved based on the objective data correlation to obtain the weights. Then, the time weighting factor is introduced to adjust the role of different time periods. Finally, the dynamic weights are combined with the subjective weights to obtain the final weights. The evaluation results include the capability value of robots, the capability fluctuation information, and the analysis results of relevant factors affecting sustainable manufacturing capability of ICRs, which will be used for ICRs composition optimal-selection and the next stage of production and scheduling of ICRs systems.

\section{DYNAMIC ASSESSMENT METHOD BASED ON CORRELATION MODEL}

\subsection{ICRs Sustainable Manufacturing Capability Correlation Model}

\subsubsection{Ontology-based Correlation Relationships Description Model}

The original evaluation indicator information of the ICRs comes from the direct measurement data of the production process, the manufacturing capability service correlation relationships are not included. The service correlation information is distributed in the form of textual data in various manufacturing companies and various stage of manufacturing process. In addition, the data correlation information obtained with mining needs to be uniformly stored for subsequent related calculations. Based on ontology, this paper formalizes the above three types of ICRs correlations and establishes the correlation model of sustainable manufacturing capability.

The ontology of the correlation model is constructed by combining the sustainable manufacturing capability evaluation indicators, as described in Eq. (12):

Capability_Info $=($ TargetNeeds_Quality, TargetNeeds_Time,

TargetNeeds_Cost,TargetNeeds_EnergyConsumption,

TargetNeeds_EnvironmentalProtection,

TargetNeeds_RecyclingBenefits,

CompositionCorrelationRelationships_Info)

Then, the Protégé 5.0 ontology editor is used to describe the sustainable manufacturing capability of the ICRs, as shown in Fig5. 


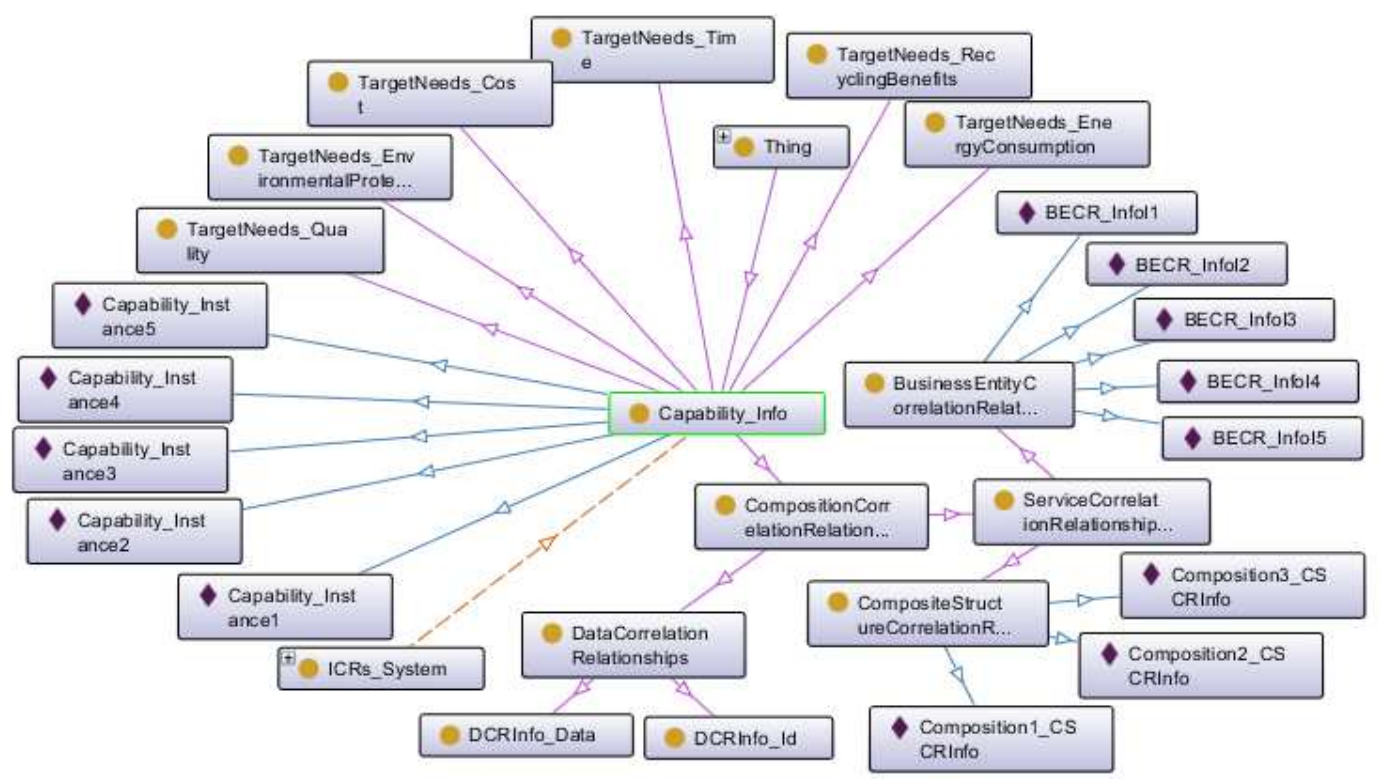

Fig5. The Structure of Manufacturing Capability Information Ontology

\subsubsection{Service Correlation Processing of Indicator Raw Data}

According to the type of service correlation relationship, the service correlation processing can be further divided into the following two aspects:

(1) Indicator correlation processing for composite structure correlation relationships

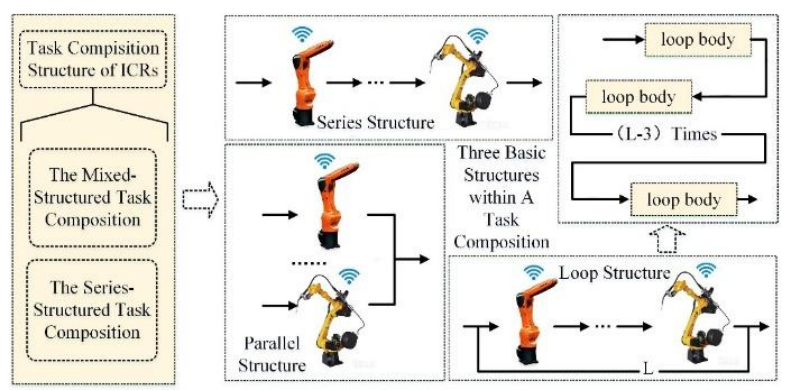

Fig6. The Overall Structure of ICRs Composition

From the perspective of the overall composition structure, the ICRs composition for multi-resource service request tasks can generally be divided into two forms as shown in Fig6: the series-structured task composition and the mixedstructured task composition. The seriesstructured task composition consists of a series of ICRs services with end-to-end rules, and any adjacent robots form a basic series structure. The mixed-structured type task composition is a complex composition structure formed by mixing three basic composition structures (series structure, parallel structure, and loop structure). Among the three basic structures, the loop structure can be represented as a series of multiple loop bodies. In summary, for all types of
ICRs task compositions, they can be expressed in the form of the basic series structure and the basic parallel structure. Therefore, the disassembly results of the ICRs composition structure are defined as follows:

$$
\begin{aligned}
& I C R s_{-} C_{\text {structure }}=\{I(s, 1):\{T(s, 1)\} ; \cdots ; I(s, i):\{T(s, i)\} ; \\
& I\left(p, 1, k_{1}\right):\left\{\{T(p, 1,1)\} ; \cdots ;\left\{T\left(p, 1, k_{1}\right)\right\}\right\} ; \cdots ; \\
& \left.I\left(p, j, k_{j}\right):\left\{\{T(p, j, 1)\} ; \cdots ;\left\{T\left(p, j, k_{j}\right)\right\}\right\}\right\}
\end{aligned}
$$

where $I(s, i)$ stands for the series element, namely, a child node that forms a series relation with other parts of the composition. If there is a series relationship between a parallel structure and a series element, the parallel structure as a whole is considered as a series factor. $i$ is the total number of all series factors in the robot composition. $I\left(p, j, k_{j}\right)$ stands for the parallel structure block, $j$ is the total number of parallel structures in the ICRs composite service, and $k_{j}$ is the total number of the parallel element in the parallel structure $I\left(p, j, k_{j}\right) . T(s, i)$ and $T\left(p, j, k_{j}\right)$ respectively stand for the value of the time type indicator of the series element $I(s, i)$ and the parallel element $I\left(p, j, k_{j}\right)$.

The composite structure correlation relationships will not affect the evaluation of other types of indicators except time-type indicators, and the detailed processing methods of time-type indicators are formulated as follows: 


$$
\begin{aligned}
& T^{\text {afterSC }}(s, n)=T(s, n), n=1,2, \cdots, i \\
& T^{\text {afterSC }}\left(p, h, k_{r}\right)=0, r=1,2, \cdots, n, \\
& \text { where } T\left(p, h, k_{r}\right) \neq \max \left(T\left(p, h, k_{1}\right), \cdots, T\left(p, h, k_{n}\right)\right) \\
& T^{\text {afterSC }}\left(p, n, k_{n}\right)=T\left(p, n, k_{m}\right), n=1,2, \cdots, j \\
& \text { where } T\left(p, n, k_{m}\right)=\max \left(T\left(p, n, k_{1}\right), \cdots, T\left(p, n, k_{n}\right)\right)
\end{aligned}
$$

where $T^{\text {afterSC }}(s, n)$ and $T^{\text {afterSC }}\left(p, h, k_{r}\right)$ stand for the value of the time type indicator of $I(s, n)$ and $I\left(p, h, k_{r}\right)$ after correlation processing.

(2) Indicator correlation processing for the business entity correlation relationships

There are four types of business entity correlations [10] between ICRs services: 1)the preferential cooperation correlation, for example, the ICRs service demander uses the robot A and $B$ at the same time, it will additionally enjoy certain reductions in processing costs, 2)the joint production correlation, namely, two or more robots uses the technical alliance to jointly produce and process standardized parts to form a quality advantage, 3)the benign competition correlation, the process by which two or more similar robots improve the cost performance of their services in the process of competition, 4)the vicious competition correlation, contrary to the benign competition correlation, it will lead to a decline in service cost performance. The results of the four correlations appear as three states: cooperation, competition, non-cooperation and non-competition.

Assume that the $s$ indicator of the $k$ ICRs service is $q_{k}^{s}$, and the default value of indicator $q_{k}^{s}$ is default value. There are a total of $m$ business entity correlations that affect the indicator $q_{k}^{s}$, desc $\operatorname{BEC}\left(q_{k}^{s}, m\right)$ is used to represent the $m$ correlation statement of $q_{k}^{s}$. At the same time, the symbol $\operatorname{BEC}\left(q_{k}^{s}, m\right)$ is used to describe the indicator value after the indicator changes due to the existence of the business entity correlation relationship $\operatorname{desc} \_\operatorname{BEC}\left(q_{k}^{s}, m\right)$. Finally, the business entity correlation description of the indicator $q_{k}^{s}$ is measured as follows:

$q_{k}^{s}=\left\{q\left(k_{s}, 0\right):\right.$ default value $\left.; q\left(k_{s}, 1\right): B E C\left(k_{s}, 1\right) ; \cdots ; q\left(k_{s}, m\right): B E C\left(k_{s}, m\right)\right\}$

According to the polarities of the evaluated indicators, Eq. (18) and Eq. (19) are used respectively to process the business entity correlation of the indicator data for the forwardtype indicator and the reverse-type indicator.

$q_{k}^{s} \_$val $=\max \left(q\left(k_{s}, r\right) *\right.$ whether $\left.\left(k_{s}, r\right)\right), r=0,1,2, \cdots, m(18)$
$q_{k-}^{s}$ val $=\min \left(q\left(k_{s}, r\right) *\right.$ whether $\left.\left(k_{s}, r\right)\right)$, whether $\left(k_{s}, r\right)=1, r=0,1,2, \cdots, m$

whether $\left(k_{s}, r\right)$ is a judgment symbol, which is mainly used to judge whether the business entity correlation $\operatorname{desc}{ }_{-} B E C\left(q_{k}^{s}, m\right)$ exists in the task composition and production process of the current ICRs composition to be evaluated. The specific definition is as follows:

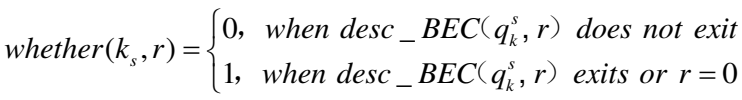

\subsubsection{Data Correlation Mining and Processing}

Data correlation mining will be carried out for each evaluation indicator in the ICRs composition to be evaluated, and the mining results will be filtered to support subsequent weight allocation based on the objective correlation information of the indicators.

The Maximal Information Coefficient $(\mathrm{MI}(\mathrm{C})$ algorithm, is proposed based on the mutual information to capture the dependency relationship between two variables in big data sets [40]. Suppose that an ordered data set consisting of two variables $D=\left\{\left(x_{i}, y_{i}\right), i=1,2, \cdots, n\right\}$ is distributed in two dimensions, the $x \times y$ grid is used to divide the data space to obtain an $x \times y$ grid $G$. Then, the MIC value of the data set $D^{G}$ is defined as follows, which represents the degree of correlation between the two variables:

$$
\begin{gathered}
\operatorname{MIC}(D)=\max _{|X| \times|Y| \leq B(n)}\left(M(D)^{x, y}\right) \\
M(D)^{x, y}=\frac{I^{\max }(D, x, y)}{\log \min \{|X|,|Y|\}} \\
I^{\max }(D, x, y)=\max _{\text {all } G}\left(\left(I\left(D^{G}\right)\right)\right) \\
I\left(D^{G}\right)=\sum_{x \in X, y \in Y} P(x, y) \log \frac{P(x, y)}{\sum_{x \in X} P(x, y) \sum_{y \in Y} P(x, y)}
\end{gathered}
$$




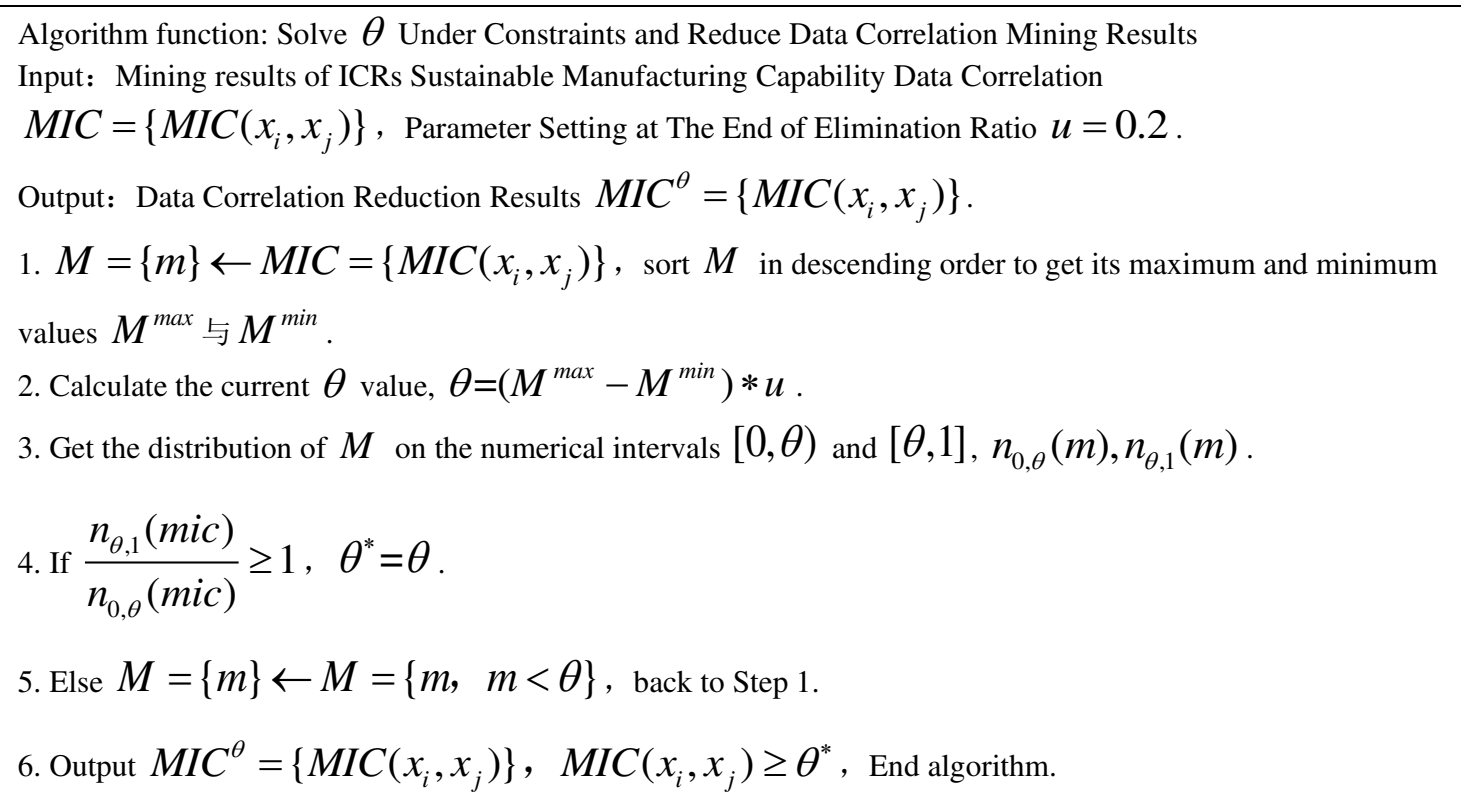

Fig7. The Pseudo-code for Solving $\theta$ and Reducing Data Correlation Results

$I\left(D^{G}\right)$ is the mutual information of $D^{G}$, and $B(n)$ (In general, $B(n)=n^{0.6}$ ) is the upper limit of the number of divided grids.

A data correlation mining method for largescale data, namely the MIC fast algorithm [41], is adopted to mine the data correlation among evaluation indicators. The data mining results of indicators $x_{i}, x_{j}$ will finally be presented in the form of $\operatorname{MIC}\left(x_{i}, x_{j}\right)$, and $\operatorname{MIC}\left(x_{i}, x_{j}\right) \in[0,1]$ . The larger the $\operatorname{MIC}\left(x_{i}, x_{j}\right)$ value, the greater the degree of correlation between the indicators $x_{i}, x_{j}$.

In order to eliminate redundant indicator data correlation information in the mining results to optimize subsequent calculations, a screening threshold $\theta$ is set for the mining results, and the part with a MIC value less than the parameter $\theta$ is reduced.

The constraint conditions on $\theta$ is set by combining the elimination ratio at the end and the distribution of $\operatorname{MIC}\left(x_{i}, x_{j}\right)$ data on the interval $[0,1]$ to obtain the value of $\theta$ to obtain the data correlation reduction results. The pseudo code of the algorithm is shown in Fig7.

\subsection{Dynamic Comprehensive Evaluation and Composition Optimal-Selection based on Correlation Model}

\subsubsection{Weight Allocation based on Improved ANP Method}

The traditional ANP method based on the objective data correlation relationship between indicators is improved in this study to enhance the objectivity of the weighting coefficient solution process. The specific operation of the improved ANP method is as follows:

Step1: Based on the mining results of indicator data correlations, combined with the expert experience and judgment, the interdependence and feedback relationships of indicators and indicators sets are analysed. The network structure of the ICRs sustainable manufacturing capability indicator is constructed, as shown in Fig8.

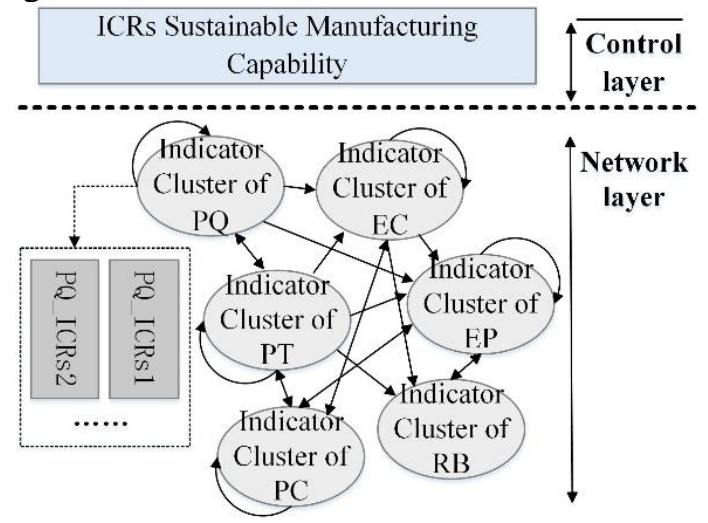

Fig8. The Network Structure of Indicators 
Step 2: By calculating the difference between two MIC values of two indicators to be compared under the same criterion, construction of the judgment matrix is completed using the calculation results. The objective data correlations between assessment indicators are used to replace the subjective judgment of the relative importance of paired indicators based on the experience of experts in the traditional ANP method. Concretely, suppose indicator $e_{r}$ is taken as the criterion indicator, $e_{r}$ is an indicator in another indicator set. In addition, the judgment matrix of the indicator cluster $E_{t}$ under indicator $e_{r} \quad$ is denoted as $A^{r / E t}=\left(a_{i j}^{r / E t}\right), a_{j i}^{r / E t}=1 / a_{i j}^{r / E t}, i, j=1,2, \cdots, t$. Then two indicators in cluster $E_{t}$, namely $e_{i}$ and $e_{j}$, are taken as examples to illustrate the specific process of establishing the judgment matrix $A^{r / E t}$ in three different situations:

$a_{i j}^{\prime \prime E}=\frac{\operatorname{MIC}\left(e_{r}, e_{i}\right)-\operatorname{MIC}\left(e_{r}, e_{j}\right)}{\max \left(\operatorname{MIC}\left(e_{n}, e_{m}\right)\right)-\min \left(\operatorname{MIC}\left(e_{n}, e_{m}\right)\right)} \times n, \operatorname{MIC}\left(e_{r}, e_{i}\right)>\operatorname{MIC}\left(e_{r}, e_{j}\right)$

$a_{i j}^{r \mid E t}=1, \operatorname{MIC}\left(e_{r}, e_{i}\right)=\operatorname{MIC}\left(e_{r}, e_{j}\right)$

$a_{i j}^{n t I}=\frac{\operatorname{MIC}\left(e_{r}, e_{j}\right)-\operatorname{MIC}\left(e_{r}, e_{i}\right)}{\max \left(\operatorname{MIC}\left(e_{n}, e_{m}\right)\right)-\min \left(\operatorname{MIC}\left(e_{n}, e_{m}\right)\right)} \times n, \operatorname{MIC}\left(e_{r}, e_{i}\right)<\operatorname{MIC}\left(e_{r}, e_{j}\right)$

where $e_{n}$ and $e_{m}$ are any indicators in any indicator clusters of assessment target, $1 \square n$ is the selected scale. $\operatorname{MIC}\left(e_{n}, e_{m}\right)$ is the MIC value of the indicators $e_{n}$ and $e_{m}$.

Step 3: Optimize the $a_{i j}^{r / E t}$ obtained above to make it more evenly distributed:

$a_{i j}^{r I E t}=1$, if $\frac{\left|\operatorname{MIC}\left(e_{r}, e_{i}\right)-\operatorname{MIC}\left(e_{r}, e_{j}\right)\right|}{\max \left(\operatorname{MIC}\left(e_{n}, e_{m}\right)\right)-\min \left(\operatorname{MIC}\left(e_{n}, e_{m}\right)\right)}<\frac{1}{n}$

Step 4: The super-matrix is constructed, each column of the super-matrix is normalized, and the relative ranking vector, namely, the weight coefficient of the indicator, is finally calculated.

\subsubsection{Dynamic Comprehensive Assessment of ICRs Sustainable Manufacturing Capability}

Potential data correlations between evaluation indicators are used to calculate the weights of indicators based on objective information. However, for the assessment of the sustainable manufacturing capability of the ICRs, it is not enough to simply assign the weights of the indicators based on the objective characteristics of the data.

Since different users or decision makers in the system attach different importance to different indicators and different targets, it is necessary to introduce subjective weight to adjust the above weight coefficient to make the evaluation results reflect the subjective preference of decision makers or cloud users as much as possible, so as to make the final evaluation results more comprehensive and easy to accept.

In order to simplify the calculation of the evaluation process and reflect the subjective information of decision makers, this paper uses the AHP method [29] to determine the subjective weight coefficient of each sustainable manufacturing capability indicator.

In order to comprehensively and accurately grasp the state fluctuations of ICRs individual and ICRs compositions due to changes in their own state, environment and other factors over a period of time, the time weighting factors $v=\left(v_{1}, v_{2}, \cdots, v_{N}\right)$ for $N$ different time periods $T_{1}, T_{2}, \cdots, T_{N}$ is determined.

To solve the time weight vector $v=\left(v_{1}, v_{2}, \cdots, v_{N}\right)$, the time-degree factor $\lambda=\sum_{k=1}^{N} \frac{N-k}{N-1} v_{k}$ is introduced, which is used to characterize the preference degree for time series. When $\lambda$ takes different values within its range, the time weight vector $v$ also changes. In order to select a $v$ solution with the least fluctuation from the many solution sets of time weight vectors, the minimal variability method [42] is adopted as follows: 


$$
\left\{\begin{array}{l}
\min \left(\frac{1}{N} \sum_{k=1}^{N} v_{k}^{2}-\frac{1}{N^{2}}\right) \\
\text { s.t. } \lambda=\sum_{k=1}^{N} \frac{N-k}{N-1} v_{k} \\
\sum_{k=1}^{N} v_{k}=1, v_{k} \in[0,1], k=1,2, \cdots, N
\end{array}\right.
$$

A dynamic comprehensive assessment of the ICRs sustainable manufacturing capability is as follows:

$$
\begin{gathered}
C=\sum_{l=1}^{N}\left(\sum_{r=1}^{n} w_{r}^{l} x_{r}\left(T_{l}\right)\right) * v_{l} \\
w_{r}^{l}=\frac{w^{c}{ }_{r} w^{s}{ }_{r}}{\sum_{j=1}^{n} w^{c}{ }_{j} w^{s}{ }_{j}}
\end{gathered}
$$

where $x_{r}\left(T_{l}\right)$ is the $r$ indicator value of the ICRs to be evaluated in the $T_{l}$ period. The vector $w^{c}=\left(w_{1}^{c}, w_{2}^{c}, \cdots, w_{n}^{c}\right)$ is the indicator weight obtained based on the objective data correlations between the evaluation indicators, and $w^{s}=\left(w_{1}^{s}, w_{2}^{s}, \cdots, w_{n}^{s}\right)$ is the subjective weight coefficient obtained by the AHP method.

\subsubsection{Composition Optimal-Selection}

The bee algorithm $[43,44]$ is adopted to realize the ICRs composition optimal-selection, and the Pareto solution is introduced to achieve multiobjective equilibrium.

Due to the complex and changeable environment of the ICRs system, the task request of ICRs service demander, the processing process of the robot to complete the task and the distribution of robot resources are all in dynamic change, a mathematical description of the composition optimal-selection problem of ICRs is presented and its optimal-selection environment is simplified reasonably.

On the premise of meeting the corresponding minimum requirements of decision makers for six aspects of sustainable manufacturing capability, in order to find composition solutions that meet the best manufacturing capability and the lowest energy consumption, two objective functions and six constraints to construct a mathematical model of the ICRs composition optimal-selection are defined in Eq. (32), where $T$ represents the processing time, $Q$ represents the processing quality, $C$ represents the processing cost, EP represents the environmental protection, $E C$ represents the energy consumption, $R$ represents the recycling benefits, and $S C$ represents the sustainable manufacturing capability of the ICRs.

$$
\left\{\begin{array}{c}
\max Y=[S C, 1 / E C] \\
\text { s.t. } T \leq T_{\max } \\
Q \geq Q_{\min } \\
C \leq C_{\max } \\
E P \leq E P_{\max } \\
R \geq R_{\min } \\
E C \leq E C_{\max }
\end{array}\right.
$$

\section{CASE STUDY AND RESULT ANALYSIS}

\subsection{Case Description}

Taking the part assembly process as an example for the research scenario, a certain part assembly task has been decomposed into five subprocessing tasks. The decomposition plan is shown in Fig9. In the cloud platform, each subtask such as the sub-task $p_{i}$ has 2 to 3 robot equipment services that meet its functional requirements to form an ICRs candidate set for the sub-task $p_{i} \leftarrow\left\{r_{i}^{1}, r_{i}^{2}, r_{i}^{3}\right\}$. The function information of each robot to perform tasks is shown in Table 5. An arbitrary robot service is selected from each ICRs candidate set to complete the execution of the corresponding subprocessing task, then a total of $C_{3}^{1} * C_{2}^{1} * C_{2}^{1} * C_{3}^{1} * C_{2}^{1}=72 \quad$ composition solutions can be formed.

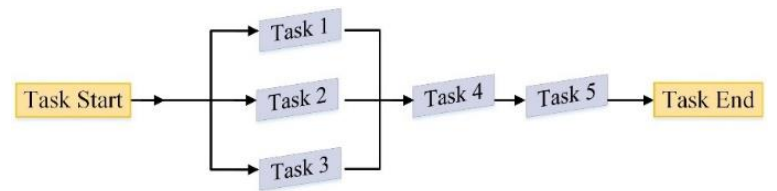

Fig9. Assembly Task Decomposition Planning

Table 5. Robot Equipment Function Matching Details

\begin{tabular}{cccccc}
\hline Sub-task Ordinal & 1 & 2 & 3 & 4 & 5 \\
\hline Robot & $\left\{r_{1}^{1}, r_{1}^{2}, r_{1}^{3}\right\}$ & $\left\{r_{2}^{1}, r_{2}^{2}\right\}$ & $\left\{r_{3}^{1}, r_{3}^{2}\right\}$ & $\left\{r_{4}^{1}, r_{4}^{2}, r_{4}^{3}\right\}$ & $\left\{r_{5}^{1}, r_{5}^{2}\right\}$ \\
\hline
\end{tabular}


The six indicators reflecting the overall sustainable manufacturing capability of the ICRs composition are recorded as the processing time $t$, the processing quality $q$, the processing cost $c$, the environmental protection $e p$, the energy consumption $e c$ and the recycling benefits $r$. In addition, the 15 sustainable manufacturing capability indicators of assembly robot equipment are the response time $t_{1}$, the auxiliary time $t_{2}$, the assembly time $t_{3}$, maintenance cost $c_{1}$, labor cost $c_{2}$, equipment depreciation $c_{3}$, dimensional accuracy $q_{1}$, mutual positional accuracy $q_{2}$, relative motion accuracy $q_{3}$, contact accuracy $q_{4}$, noise pollution $e p_{1}$, wastes $e p_{2}$, recycling benefits $r$, equipment energy consumption $e c_{1}$, auxiliary equipment energy consumption $e c_{2}$. Taking one of the robot task composition schemes ICRs $C_{1} \leftarrow\left\{r_{1}^{1}, r_{2}^{1}, r_{3}^{1}, r_{4}^{1}, r_{5}^{1}\right\}$ as an example, the specific evaluation steps are described as follows:

Step 1: Obtain the historical manufacturing task information for composition $I C R s C_{1}$. The Eq. (2) to Eq. (4) are used to quantify the qualitative indicators to form a numerical historical manufacturing data of the composition.

Step 2: Based on the service correlation description model, the service correlation processing is performed on the evaluation indicators raw data. One set of the raw data of the various indicators to be evaluated for robot equipment $r_{1}^{1}, r_{2}^{1}, r_{3}^{1}, r_{4}^{1}, r_{5}^{1}$ in $I C R s C_{1}$ is shown in Table 6, and the corresponding indicators data that has undergone service correlation processing are shown in Table 7.

Step 3: Standardize the data of all indicators after service correlation processing according to Eq. (5) to Eq. (8), and then select the last six time series $\left(T_{1}, T_{2}, T_{3}, T_{4}, T_{5}, T_{6}\right)$ to build a time series data table of indicator data. The MIC fast algorithm is used to perform data correlation mining on multiple manufacturing task data in the above six time periods, and the $\theta$ value is determined based on the mining results (the value of $\theta$ on the time period $T_{1}$ is 0.276 ). Then, the initial mining information is filtered, and finally the data correlation relationships between the indicators of $I C R s C_{1}$ under six different time series is obtained. Taking the time period of $T_{1}$ as an example, the mining results of some sustainable manufacturing capability indicator correlation relationships are shown in Table 8.

Step 4: Based on the above objective data correlation and the improved ANP method, the weight coefficients of sustainable manufacturing capability indicator are allocated to obtain an indicator weight matrix $w^{T 1}=\left(w_{-} r_{1}^{1}, w_{-} r_{2}^{1}, w_{-} r_{3}^{1}, w_{-} r_{4}^{1}, w_{-} r_{5}^{1}\right) \quad$ of ICRs composition ICRs $C_{1}$ with a size of $5 \times 15$ in the $T_{1}$. Partial weights are shown in Table 9.

Step 5: The AHP method is used to obtain the subjective weight coefficients of evaluation indicators. Partial subjective weights are shown in Table 10.

Step 6: The two weighting factors obtained above are integrated and normalized according to Eq. (31) to serve as the static comprehensive weighting of the ICRs composition ICRs $C_{1}$ in the time period of $T_{1}$. Then, the same steps are used to obtain the static comprehensive weight of evaluation indicator in time period of $T_{2}, T_{3}, T_{4}$ $, T_{5}, T_{6}$.

Step 7: The time weight is calculated by Eq. (29), take $\lambda=0.3$, and get the time weight vector of $T_{1}, T_{2}, T_{3}, T_{4}, T_{5}, T_{6}$ as $v=(0.003,0.009,0.025,0.075,0.224,0.664)$.

Table 6. The Raw Value of Indicators

\begin{tabular}{ccccccccc}
\hline Indicator & $t_{1}$ & $t_{2}$ & $t_{3}$ & $c_{1}$ & $c_{2}$ & $c_{3}$ & $q_{1}$ & $q_{2}$ \\
\hline$r_{1}^{1}$ & 3.5 & 5.2 & 12.5 & 13.2 & 22.5 & 6.3 & \pm 0.20 & \pm 0.15 \\
$r_{2}^{1}$ & 2.8 & 0 & 9.5 & 8.8 & 17.3 & 5.8 & \pm 0.20 & \pm 0.25 \\
$r_{3}^{1}$ & 3.2 & 0 & 6.7 & 5.6 & 13.1 & 5.3 & \pm 0.25 & \pm 0.30 \\
$r_{4}^{1}$ & 3 & 6.7 & 13.8 & 15.6 & 28 & 7.2 & \pm 0.15 & \pm 0.15
\end{tabular}




\begin{tabular}{|c|c|c|c|c|c|c|c|}
\hline$r_{5}^{1}$ & 3.6 & 10.3 & 7.5 & 18.3 & 32 & 6.5 & \pm 0.20 \\
\hline Indicator & $q_{3}$ & $q_{4}$ & $e p_{1}$ & $e p_{2}$ & $r$ & $e c_{1}$ & $e c_{2}$ \\
\hline$r_{1}^{1}$ & \pm 0.25 & \pm 0.20 & 2.3 & 3.1 & 8 & 35.2 & 17.8 \\
\hline$r_{2}^{1}$ & \pm 0.15 & \pm 0.30 & 2.5 & 3.5 & 6.5 & 21.3 & 9.8 \\
\hline$r_{3}^{1}$ & \pm 0.10 & \pm 0.15 & 2.5 & 2.8 & 7.3 & 15.8 & 13.6 \\
\hline$r_{4}^{1}$ & \pm 0.20 & \pm 0.20 & 2.7 & 5.4 & 5 & 39.2 & 13 \\
\hline$r_{5}^{1}$ & \pm 0.20 & \pm 0.25 & 3.5 & 4.6 & 3 & 30 & 14.6 \\
\hline
\end{tabular}

Table 7. The Value of Processed indicator data

\begin{tabular}{ccccccccc}
\hline Indicator & $t_{1}$ & $t_{2}$ & $t_{3}$ & $c_{1}$ & $c_{2}$ & $c_{3}$ & $q_{1}$ & $q_{2}$ \\
\hline$r_{1}^{1}$ & 3.5 & 5.2 & 12.5 & 10.6 & 18 & 6.3 & \pm 0.10 & \pm 0.15 \\
$r_{2}^{1}$ & 0 & 0 & 0 & 7.9 & 15.6 & 5.8 & \pm 0.20 & \pm 0.25 \\
$r_{3}^{1}$ & 0 & 0 & 0 & 5.0 & 11.8 & 5.3 & \pm 0.25 & \pm 0.30 \\
$r_{4}^{1}$ & 1.5 & 6.7 & 13.8 & 12.5 & 22.4 & 7.2 & \pm 0.10 & \pm 0.15 \\
$r_{5}^{1}$ & 3.6 & 10.3 & 7.5 & 18.3 & 32 & 6.5 & \pm 0.15 & \pm 0.15 \\
\hline Indicator & $q_{3}$ & $q_{4}$ & $e p_{1}$ & $e p_{2}$ & $r$ & $e c_{1}$ & $e c_{2}$ & \\
\hline$r_{1}^{1}$ & \pm 0.15 & \pm 0.15 & 2.3 & 3.1 & 8 & 35.2 & 17.8 & \\
$r_{2}^{1}$ & \pm 0.15 & \pm 0.30 & 2.5 & 3.5 & 7.8 & 21.3 & 6.2 & \\
$r_{3}^{1}$ & \pm 0.10 & \pm 0.15 & 2.5 & 2.8 & 8.8 & 15.8 & 9.1 & \\
$r_{4}^{1}$ & \pm 0.10 & \pm 0.20 & 2.7 & 5.4 & 5 & 39.2 & 13 & \\
$r_{5}^{1}$ & \pm 0.10 & \pm 0.20 & 3.5 & 4.6 & 3 & 30 & 14.6 &
\end{tabular}

Step 8: The comprehensive sustainable manufacturing capability $S C^{1}$ of the ICRs composition $I C R s C_{1}$ is calculated through Eq. (30). The information of processing time $S C^{1}-t$, processing cost $S C^{1}-c$, processing quality $S C^{1}-q$, environmental protection $S C^{1}-e p$, recycling benefits $S C^{1}-r$, and energy consumption $S C^{1}-e c$ are respectively obtained as shown in Table 11.

Step 9: Through the same steps described above, other composition schemes under this processing task are evaluated separately, and then the composition optimal-selection is made based on the sustainable manufacturing capability information of each ICRs composition. In combination with the actual manufacturing needs of the user, the goal of the optimal-selection is to find an ICRs composition service with the highest comprehensive value of sustainable manufacturing capability and the lowest energy consumption under all constraints of Eq. (32). The reverse indicators have been forwarded during the evaluation process, the constraints on the six sustainable manufacturing capabilities of the ICRs compositions are set as follows: $t>0.115, c>0.12, q>0.128, e p>0.07$, $r>0.04, \quad e c>0.13$. Multi-objective optimization is performed on the above 72 ICRs composition schemes using the enumeration method to obtain the solution space under the above constraint conditions, including 12 groups of Pareto solutions and 39 other solutions, as shown in Fig10.

Step 10: Based on the evaluation results, the above 12 groups of ICRs composition Pareto solutions are sorted respectively by $S C, t, c$, $q, e c$, providing users with flexible alternatives to deal with some changes in the task execution process. Table 12 shows the first three ICRs compositions for each sorting requirement. 
Table 8. Partial Data Correlation Mining Results

\begin{tabular}{cccc}
\hline Correlations & Indicator-A & Indicator-B & MIC Value \\
\hline 1 & $t_{3}-r_{4}^{1}$ & $e c_{1}-r_{4}^{1}$ & 0.899 \\
2 & $q_{1}-r_{4}^{1}$ & $q_{1}-r_{5}^{1}$ & 0.532 \\
3 & $q_{1}-r_{1}^{1}$ & $q_{1}-r_{4}^{1}$ & 0.515 \\
4 & $t_{3}-r_{2}^{1}$ & $q_{2}-r_{2}^{1}$ & 0.622 \\
5 & $t_{3}-r_{1}^{1}$ & $e c_{1}-r_{1}^{1}$ & 0.874 \\
6 & $e p_{2}-r_{5}^{1}$ & $c_{2}-r_{5}^{1}$ & 0.377 \\
7 & $q_{1}-r_{1}^{1}$ & $q_{1}-r_{5}^{1}$ & 0.403 \\
8 & $q_{3}-r_{5}^{1}$ & $t_{1}-r_{1}^{1}$ & 0.371 \\
9 & $q_{2}-r_{3}^{1}$ & $q_{3}-r_{3}^{1}$ & 0.779 \\
$\ldots$ & $\ldots$ & $\ldots$ & $\ldots$ \\
352 & $r_{-} r_{3}^{1}$ & $r$ & $r_{1}^{1}$ \\
\hline
\end{tabular}

Table 9. The weight of the indicator in $r_{1}^{1}$ in time period of $T_{1}$

\begin{tabular}{ccccccccc}
\hline Indicator & $t_{1}$ & $t_{2}$ & $t_{3}$ & $c_{1}$ & $c_{2}$ & $c_{3}$ & $q_{1}$ & $q_{2}$ \\
Weights & 0.0131 & 0.0174 & 0.0176 & 0.0159 & 0.0133 & 0.0123 & 0.0168 & 0.0179 \\
\hline Indicator & $q_{3}$ & $q_{4}$ & $e p_{1}$ & $e p_{2}$ & $r$ & $e c_{1}$ & $e c_{2}$ \\
Weights & 0.0159 & 0.0166 & 0.0145 & 0.0134 & 0.0143 & 0.0124 & 0.0126 \\
\hline
\end{tabular}

Table 10. The subjective weight of the indicator in $r_{1}^{1}$

\begin{tabular}{ccccccccc}
\hline Indicator & $t_{1}$ & $t_{2}$ & $t_{3}$ & $c_{1}$ & $c_{2}$ & $c_{3}$ & $q_{1}$ & $q_{2}$ \\
Weights & 0.0092 & 0.0104 & 0.0132 & 0.0135 & 0.0143 & 0.0122 & 0.0141 & 0.0133 \\
\hline Indicator & $q_{3}$ & $q_{4}$ & $e p_{1}$ & $e p_{2}$ & $r$ & $e c_{1}$ & $e c_{2}$ & \\
Weights & 0.0127 & 0.0131 & 0.0140 & 0.0125 & 0.0135 & 0.0280 & 0.0231 & \\
\hline
\end{tabular}

Table 11. The Sustainable Manufacturing Capability Information of $I C R s C_{1}$

\begin{tabular}{cccccccc}
\hline Capability & $S C^{1}$ & $S C^{1}-t$ & $S C^{1}-c$ & $S C^{1}-q$ & $S C^{1}-e p$ & $S C^{1}-r$ & $S C^{1}-e c$ \\
\hline Value & 0.6652 & 0.1265 & 0.1299 & 0.1356 & 0.0803 & 0.0474 & 0.1455 \\
\hline
\end{tabular}

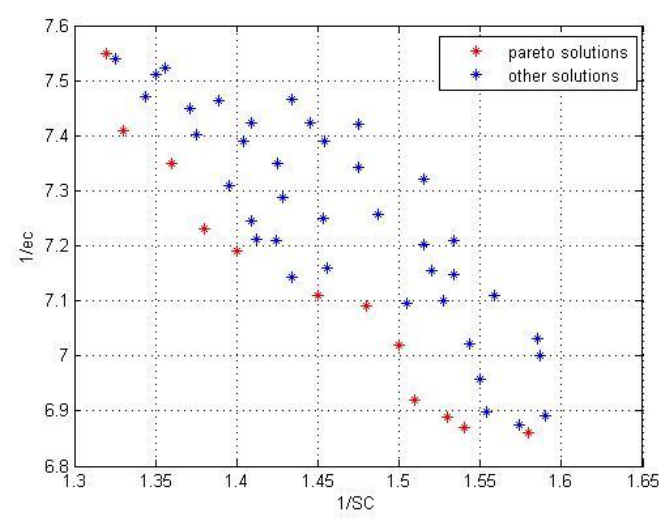

Fig10. The Solution Space under Enumeration Method 
Table 12. Sorting results of Pareto solution of ICRs composition under different needs

\begin{tabular}{cccc}
\hline $\begin{array}{c}\text { Sorting } \\
\text { Criteria }\end{array}$ & 1 & 2 & 3 \\
\hline$S C$ & $r_{1}^{2}, r_{2}^{2}, r_{3}^{1}, r_{4}^{3}, r_{5}^{1}$ & $r_{1}^{3}, r_{2}^{2}, r_{3}^{1}, r_{4}^{1}, r_{5}^{1}$ & $r_{1}^{3}, r_{2}^{1}, r_{3}^{1}, r_{4}^{3}, r_{5}^{2}$ \\
$t$ & $r_{1}^{1}, r_{2}^{1}, r_{3}^{1}, r_{4}^{3}, r_{5}^{1}$ & $r_{1}^{3}, r_{2}^{1}, r_{3}^{1}, r_{4}^{3}, r_{5}^{2}$ & $r_{1}^{2}, r_{2}^{1}, r_{3}^{1}, r_{4}^{1}, r_{5}^{2}$ \\
$c$ & $r_{1}^{3}, r_{2}^{2}, r_{3}^{1}, r_{4}^{1}, r_{5}^{1}$ & $r_{1}^{1}, r_{2}^{2}, r_{3}^{1}, r_{4}^{3}, r_{5}^{2}$ & $r_{1}^{1}, r_{2}^{1}, r_{3}^{1}, r_{4}^{1}, r_{5}^{1}$ \\
$q$ & $r_{1}^{2}, r_{2}^{2}, r_{3}^{1}, r_{4}^{3}, r_{5}^{1}$ & $r_{1}^{3}, r_{2}^{2}, r_{3}^{1}, r_{4}^{1}, r_{5}^{1}$ & $r_{1}^{3}, r_{2}^{1}, r_{3}^{2}, r_{4}^{3}, r_{5}^{2}$ \\
$e c$ & $r_{1}^{2}, r_{2}^{1}, r_{3}^{1}, r_{4}^{1}, r_{5}^{1}$ & $r_{1}^{1}, r_{2}^{2}, r_{3}^{2}, r_{4}^{3}, r_{5}^{1}$ & $r_{1}^{2}, r_{2}^{1}, r_{3}^{2}, r_{4}^{3}, r_{5}^{2}$ \\
\hline
\end{tabular}

\subsection{Comparative Analysis of Different Correlation Coefficient Algorithms in Data Correlation Mining}

In order to verify the effectiveness of the data correlation mining method, 6 sets of data with various relationships are randomly generated. Each set of data contains 3,000 data. Then three different algorithms (Pearson algorithm, Spearman algorithm and the MIC algorithm) are used to mine the data sets as shown in Fig11.

The results show that the MIC algorithm can capture a wide variety of relationships, not limited to specific function types. Namely, the universality of the MIC algorithm is superior to the other two algorithms.

\subsection{Comparative Analysis of Sustainable Manufacturing Capability under Different Evaluation Methods}

The traditional ANP method [45], the dynamic assessment method based on correlation relationships (DAESSBCR) proposed in this study, dynamic evaluation method based on error feedback [12], and FAHP-fuzzy VIKOR method [34] are respectively used to evaluate the $S C$ of ICRs composition $\operatorname{ICRs}_{1}\left(C_{1}\right)$ and the five robot Individuals $r_{1}^{1}, r_{2}^{1}, r_{3}^{1}, r_{4}^{1}, r_{5}^{1}$, as shown in Fig 12 (in order to make the results more intuitive, the $S C$ values in four methods are reduced by the same proportion).
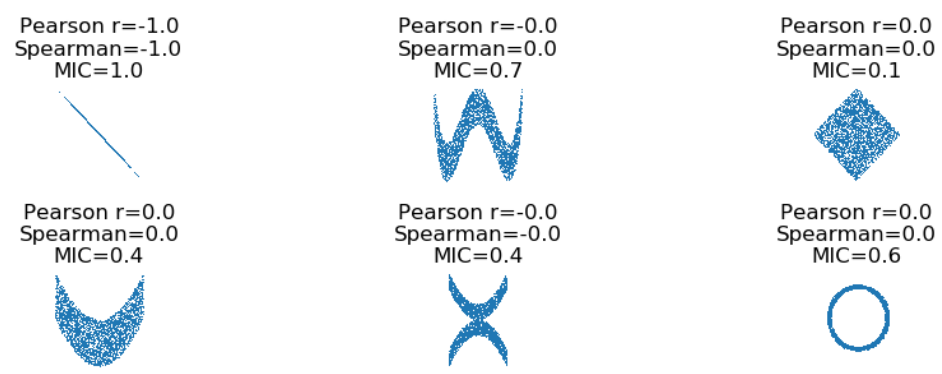

Fig11. The Results of Three Data Correlation Mining Methods

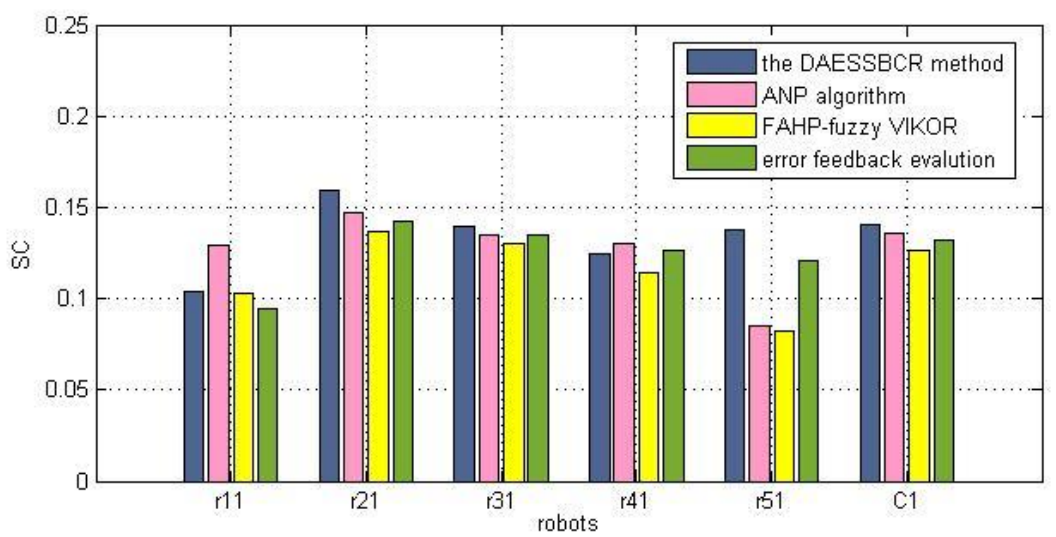

Fig12. The Sustainable Manufacturing Capability under Different Evaluation Methods 
In Fig12, for the evaluation results of the ANP method and FAHP-fuzzy VIKOR method, the $S C$ of the robot $r_{5}^{1}$ is significantly lower than that of the other four robot individuals. The ranking results $r_{2}^{1}>r_{3}^{1}>r_{5}^{1}>r_{4}^{1}>r_{1}^{1}$ obtained by using the DAESSBCR method are basically consistent with the ranking results $r_{2}^{1}>r_{3}^{1}>r_{4}^{1}>r_{5}^{1}>r_{1}^{1}$ of the error feedback evaluation.

In order to further study the consistency of the ranking results of the DAESSBCR method and the error feedback evaluation, the DAESSBCR method and the error feedback evaluation method are respectively used to obtain the $S C$ change curve of $r_{1}^{1}, r_{2}^{1}, r_{3}^{1}, r_{4}^{1}, r_{5}^{1}$, as shown in Fig13.

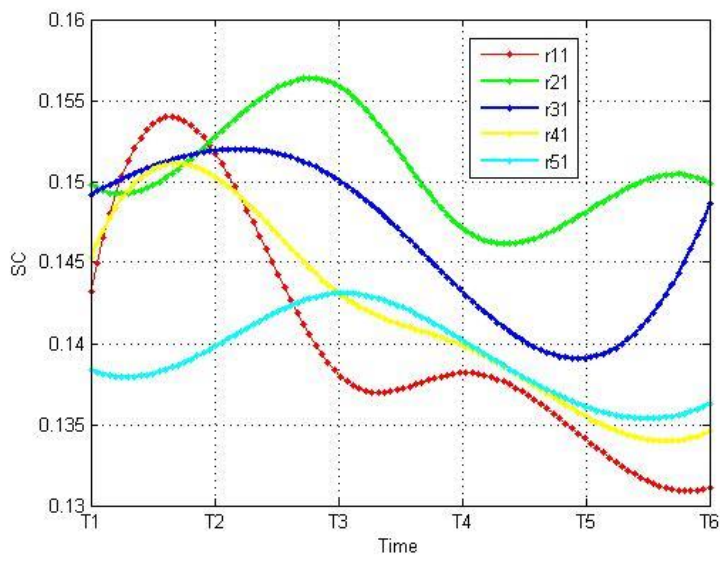

(a) The DAESSBCR Method

Fig13. The Sustainable Manufacturing Capability Change Curve under Different Methods

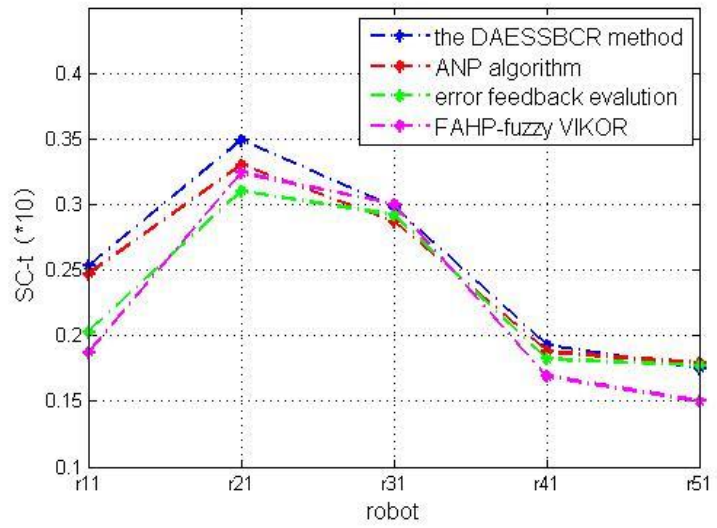

(a) Processing Time Capability

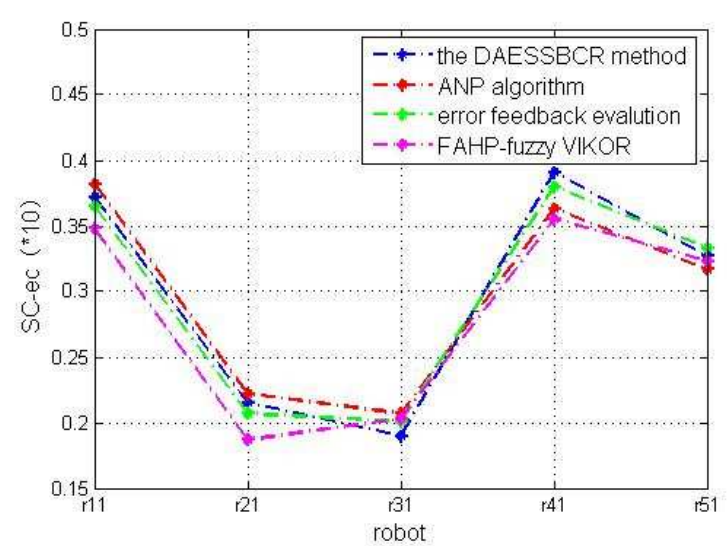

(b) Energy Consumption Capability 


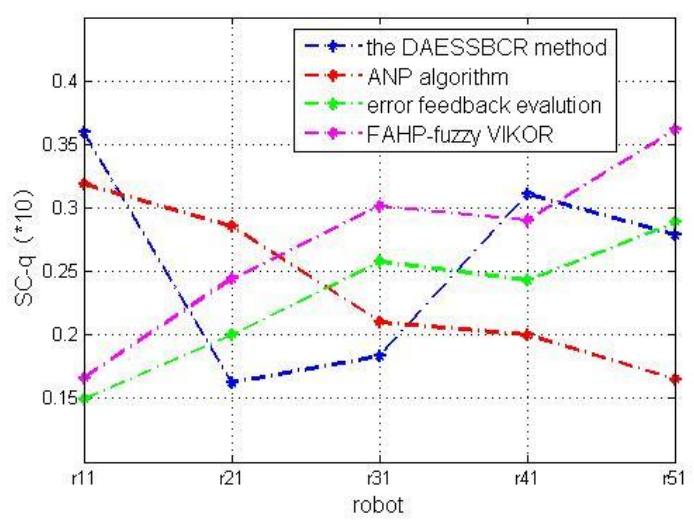

(c) Processing

Quality Capability

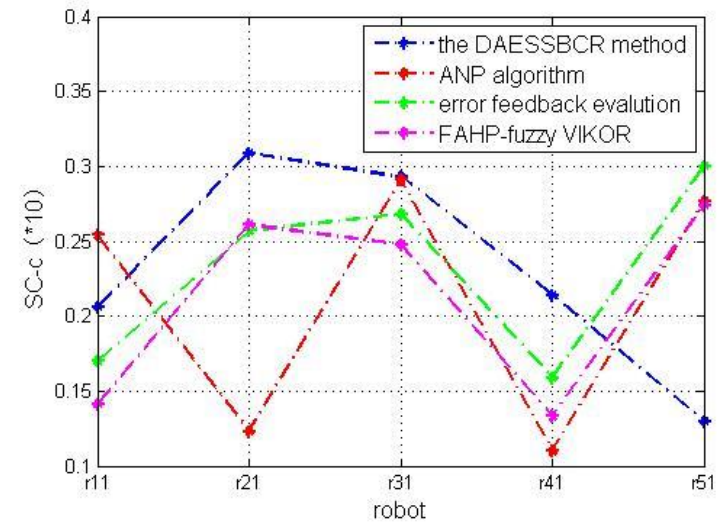

(d) Processing Cost Capability

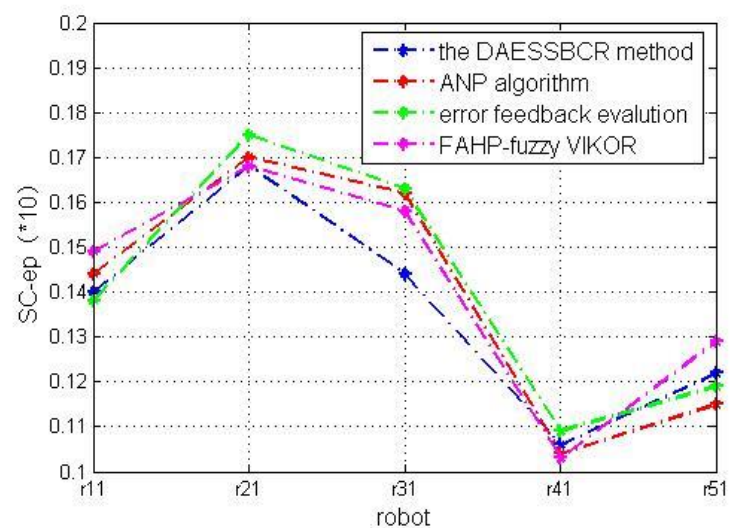

(f) Environmental Protection Capability

(e) Recycling Capability

ur Methods in Different Capability Aspects

The capability change curves of the four evaluation methods in Fig14-(a), Fig14-(b), and Fig14-(f) are consistent. The results of the four assessment methods are consistent in the aspects of processing time capability, energy consumption capability and environmental protection capability.

However, in Fig14-(c), Fig14-(d), and Fig14(e), there are significant differences in the relative ranking of robots. The FAHP-fuzzy VIKOR method, the error feedback evaluation method and the DAESSBCR method have all integrated subjective and objective weights in the evaluation process. Therefore, their relative ranking of robot manufacturing capabilities is similar. The validity of the weight allocation method in DAESSBCR, namely the improved ANP method, is verified.

But in Fig14-(c), there is still a phenomenon that the DAESSBCR method is inconsistent with the error feedback evaluation and FAHP-fuzzy VIKOR on ranking results. Judging from the evaluation process, the calculation of the comprehensive value of the robot's processing quality capability is not only related to the weight coefficient, but also the data value of indicator. Combining Table 6 and Table 7, the raw data of the processing quality related indicators of robot $r_{1}^{1}, r_{4}^{1}, r_{5}^{1}$ have been "co-optimized" to a certain extent after the service correlation processing. However, both the error feedback evaluation and FAHP-fuzzy VIKOR method directly use the raw data in the evaluation, and do not consider the positive and negative cooperative effects of the robots on the processing quality. Therefore, in the process of robot individuals forming a robot composition and performing collaborative production, the DAESSBCR method will be more in line with the actual manufacturing situation.

In addition, two dynamic evaluation methods are used to evaluate the processing time capability and energy saving capability of ICRs, as shown in Fig 15 and Fig16 (The manufacturing capability value of $C_{1}$ is reduced by the same proportion in each period). 


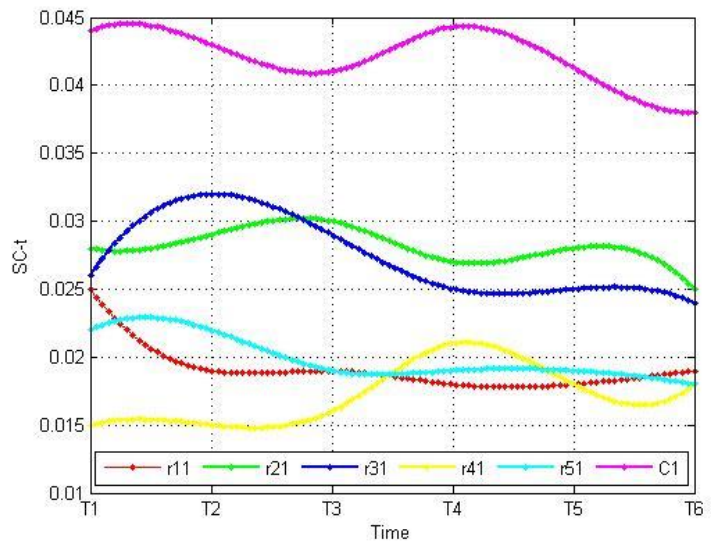

(a) The DAESSBCR Method

Fig15. The Processing Time Capability Change Curve under Different Methods

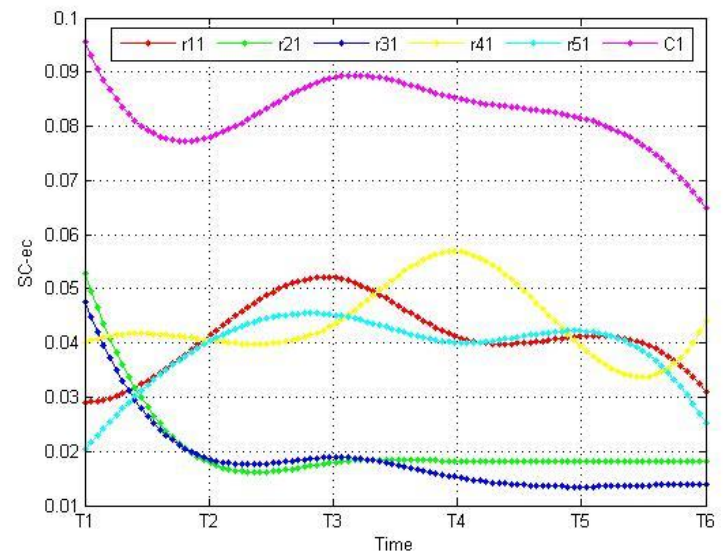

(a) The DAESSBCR Method

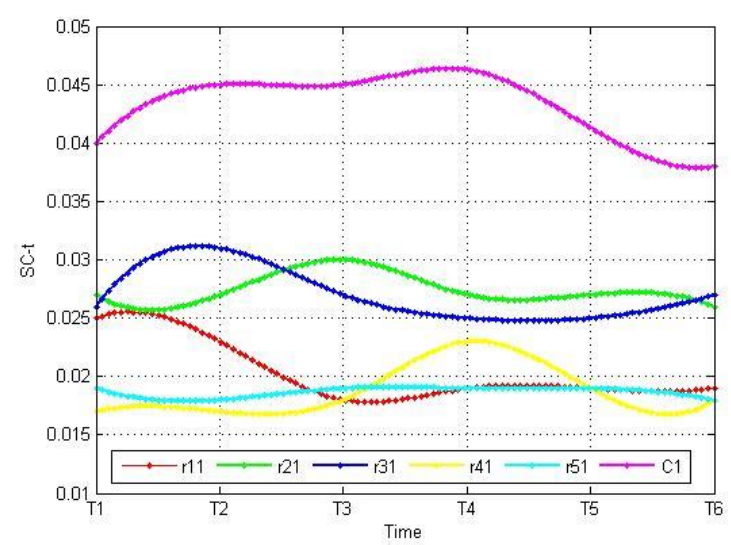

(b) Error Feedback Evaluation

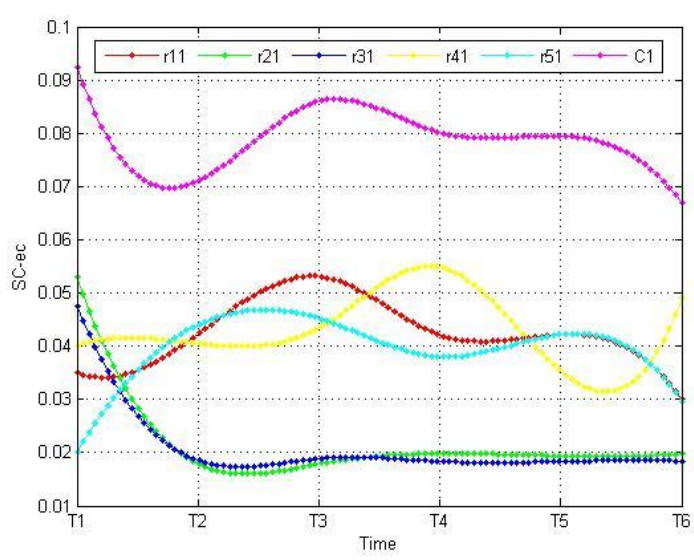

(b) Error Feedback Evaluation

Fig16. The Energy Saving Capability Change Curve under Different Methods

By comparing Fig15-(a) and Fig15-(b), the fluctuation of two processing time capability curve are different under the two methods. The processing time capability of $C_{1}$ in $T_{1}$ to $T_{2}$ period under the error feedback evaluation method is affected by the combined effects of the two robot individuals $r_{1}^{1}, r_{3}^{1}$, while the $C_{1}$ is mainly dominated by $r_{1}^{1}$ under the DAESSBCR method. A similar situation can also be found in the period from $T_{3}$ to $T_{4} . C_{1}$ in the error feedback evaluation method increase slightly under the joint influence of $r_{1}^{1}, r_{2}^{1}, r_{3}^{1}, r_{4}^{1}, r_{5}^{1}$, while $C_{1}$ in the DAESSBCR method is not significantly affected by the decline of $r_{2}^{1}, r_{3}^{1}$, but is significantly affected by the effect of $r_{4}^{1}$, and rise significantly.

Based on the combination of the sub-task execution structure of $C_{1}$ and the processing time of each robot individuals in Table 6 , the processing time capability of the composition $C_{1}$ is mainly dominated by robot $r_{1}^{1}$ and $r_{4}^{1}, r_{5}^{1}$. By comparison, the DAESSBCR method can accurately reflect the influence of individual manufacturing capability on the overall manufacturing capability of robot composition. However, the error feedback evaluation method has a slight deviation in the judgment of the relation between the composition manufacturing capability and the individual manufacturing capability.

\subsection{Comparative Analysis of ICRs Composition Optimal-Selection under Different Evaluation Methods}

The sustainable manufacturing capability evaluation results of the 72 ICRs compositions obtained under the four methods described above are used as the input of the bee algorithm [43, 44] for multi-objective optimization selection. The resulting Pareto fronts are shown in Fig17. 


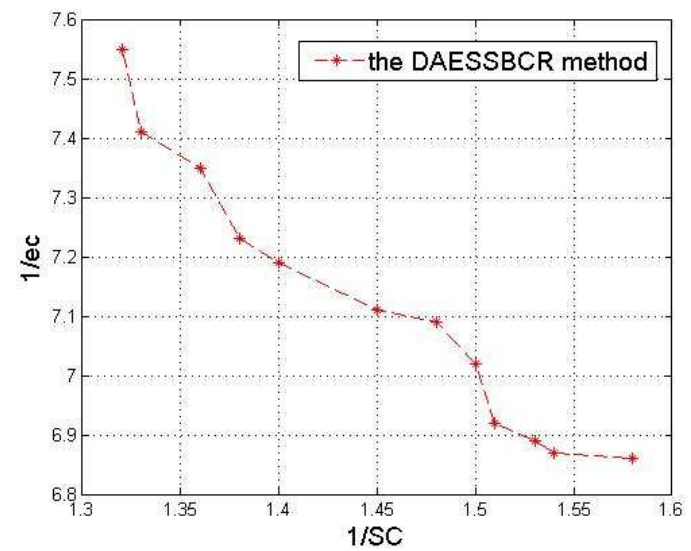

(a) The DAESSBCR Method

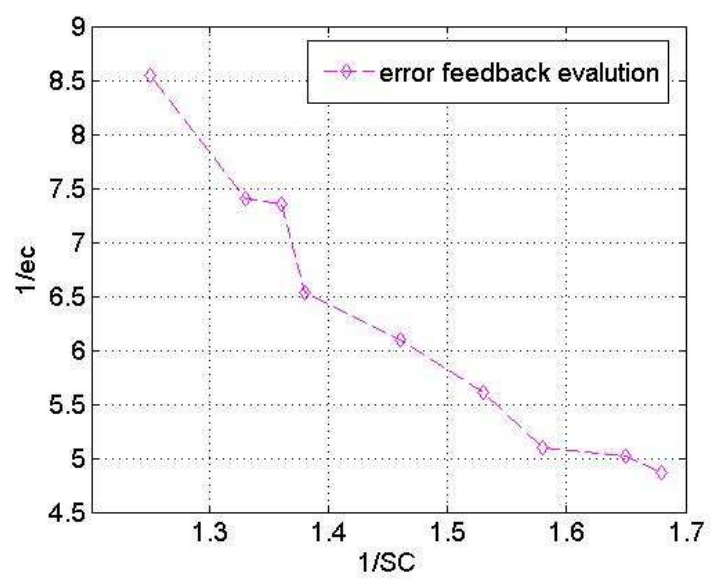

(c) Error Feedback Evaluation

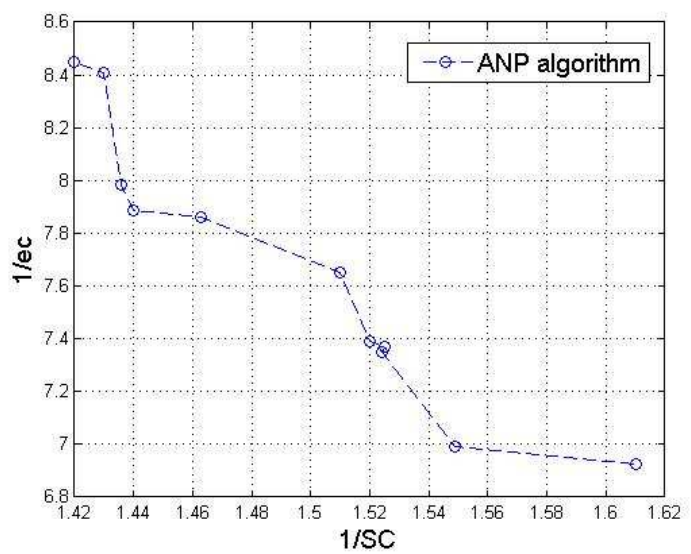

(b) ANP Algorithm

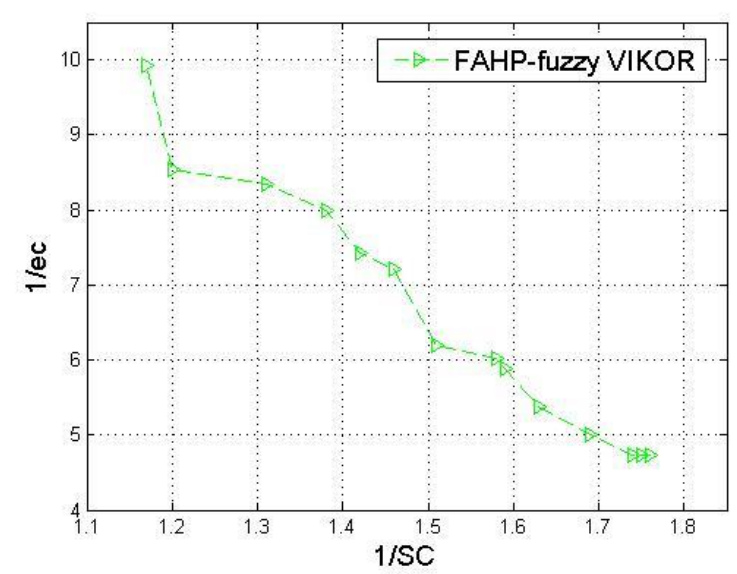

(d) FAHP-fuzzy VIKOR

Fig17. The Pareto Fronts obtained based on Different Evaluation Methods

There is no direct comparison of the superiority of the four Pareto fronts. However, according to the number of non-dominant solutions possessed by each Pareto front, the Pareto frontiers obtained based on the DAESSBCR method and FAHP-fuzzy VIKOR method have 12 and 14 non-dominant solutions respectively, while the number of non-dominant solutions obtained based on ANP method and error feedback assessment method is less than the above two methods, which are 11 and 9 respectively. From a comparative perspective, the number of schemes for ICRs composition optimal-selection provided by the DAESSBCR method is acceptable and considerable.

\section{Conclusion}

Sustainable manufacturing has become the future trends of manufacturing, and ICRs has a broad prospect in the manufacturing industry. First of all, a dynamic extensible evaluation indicator system is proposed in this paper, which can meet the needs of new types of robots in the system and the increasing personalized requirements of the indicators. Then, a correlation assessment model is established by analyzing multiple composition correlation relationships among ICRs, and a dynamic assessment method of sustainable manufacturing capability for ICRs is proposed based on the correlation relationship. Finally, some experiments of the sustainable manufacturing capability evaluation and optimalselection of the ICRs composition are carried out, the results show that the correlation evaluation model effectively considering the positive and negative cooperative effects between robots, makes the evaluation results more realistic. Compared with other evaluation work, the proposed method can more clearly reflect the organic connection between the robot individual and the ICRs composition in terms of manufacturing capability, and the evaluation results which consider the dynamic changes of manufacturing capability at different periods is more comprehensive.

In future research, the factors affecting social sustainability will be considered to establish a more comprehensive assessment indicator 
system. And how to improve the accuracy of the dynamic assessment method combined with the results of actual manufacturing process is the focus in the future work so as to predict the sustainable manufacturing capability of the ICRs based on the manufacturing capability trend.

Ethical Approval The research does not involve human participants or animals, and the authors warrant that the manuscript fulfills the ethical standards of the journal. No conflict of interest exists in the submission of this manuscript, and manuscript is approved by all authors for publication. This manuscript is an extended version of a paper presented at the 49th International Conference on Computers \& Industrial Engineering (CIE 49), Beijing, China, October 18-21, 2019.

Consent to Participate The research does not involve human participants or animals, and the authors warrant that the manuscript fulfills the ethical standards of the journal.

Consent to Publish All authors are consent to publish this manuscript in The International Journal of Advanced Manufacturing Technology.

Authors Contributions Sisi Tian proposed the method and conducted the case study, analyzed the results, and wrote the manuscript. Xiaotong Xie proposed the basic idea of the method and contributed the experimental materials. Wenjun $\mathrm{Xu}$ proposed the method design and experimental idea and also modified this manuscript. Jiayi Liu analyzed the data and conducted the case study. Xiaomei Zhang modified the structure of the manuscript and contributed the experiment works.

Funding This research is supported by National Natural Science Foundation of China (Grant No. 51775399) and the Fundamental Research Funds for the Central Universities (WUT: 2020III047).

Competing Interests The authors declare that no conflict of interest exists in the submission of this manuscript.

Availability of data and materials The data and materials of this manuscript are available from the corresponding author on reasonable request.

\section{References}

1. Yan, H., Hua, Q., Wang, Y., et al. (2017). Cloud robotics in Smart Manufacturing Environments: Challenges and countermeasures. Computers \& Electrical Engineering, 63, 56-65.

2. Liu, J., Xu, W., Zhang, J., Zhou, Z., Pham DT. (2016, June). Industrial cloud robotics towards sustainable manufacturing. ASME International Manufacturing Science and Engineering Conference (MSEC'16), USA, Blacksburg.

3. Tao, F., Zhao, D., Yefa, H., \& Zhou, Z. (2010). Correlation-aware resource service composition and optimal-selection in manufacturing grid. European Journal of Operational Research, 201(1), 129-143.

4. Ma, W., Wang, Z., Zhao, Y., et al. (2016). Optimizing services composition in cloud manufacturing based on improved ant colony algorithm. Computer Integrated Manufacturing Systems, 22(1), 113-121.

5. Jin, H., Yao, X., Chen, Y. (2015). Correlation-aware QoS modelling and manufacturing cloud service composition. Journal of Intelligent Manufacturing, 28(8), 1947-1960.

6. Farrugia, K. (2018). Industrial Robots: Accessible Opportunity. Manufacturing Engineering, 160(1), 1616.

7. Yoshio, Y. (2019). Dynamically evolving algorithm for minimizing the energy consumption of a manipulator. Artificial Life and Robotics, 24(2), 1-5.

8. Giret, A., Trentesaux, D., Prabhu, V. (2015). Sustainability in manufacturing operations scheduling: A state of the art review. Journal of Manufacturing Systems, 37, 126-140.

9. Zhang, Y., Cui, G., Deng, S. (2018). Efficient Query of Quality Correlation for Service Composition. IEEE Transactions on Services Computing, 99,1-1.

10. Guo, H., Tao, F., Zhang, L., et al. (2010). Correlationaware web services composition and QoS computation model in virtual enterprise. The International Journal of Advanced Manufacturing Technology, 51(5-8), 817-827.

11. Kootbally, Z. (2016). Industrial robot capability models for agile manufacturing. Industrial Robot: An International Journal, 43(5), 481-494.

12. Zhang, Z., Xu, W., Liu, Q., et al. (2017, June). Dynamic manufacturing capability assessment of industrial robots based on feedback information in cloud manufacturing. ASME 2017 12th International Manufacturing Science and Engineering Conference 2017, USA, Los Angeles.

13. Jin, L., Xu, W., Liu, Z., Yan, J., Zhou, Z., Pham, D. (2018, June). Knowledge Sharing and Evolution of Industrial Cloud Robotics. ASME 2018 13th International Manufacturing Science and Engineering Conference, United states, TX.

14. Du, H., Xu, W., Yao, B., Zhou, Z., Hu, Y. (2019). Collaborative Optimization of Service Scheduling for Industrial Cloud Robotics Based on Knowledge Sharing. Procedia CIRP, 83, 132-138.

15. Skinner, W. (1969). Manufacturing-missing link in corporate strategy. Harvard Business Review, 6(9), 136-145.

16. Luo, Y., Zhang, L., Tao, F., Ren, L., Liu, Y., Zhang, Z. (2013). A Modelling and Description Method of Multidimensional Information for Manufacturing Capability in Cloud Manufacturing System. International Journal of Advanced Manufacturing Technology, 69(5-8), 961-975.

17. Zhao, Y., Liu, Q., Xu, W., Wu, X., Jiang, X., Zhou, Z., Pham, D. (2017). Dynamic and unified modelling of sustainable manufacturing capability for industrial robots in cloud manufacturing. The International Journal of Advanced Manufacturing Technology, 93(5), 2753-2771. 
18. Klimchik, A., Ambiehl, A., Sébastien Garnier. (2017). Efficiency evaluation of robots in machining applications using industrial performance measure. Robotics and Computer Integrated Manufacturing, 48, 12-29.

19. Lekurwale, R.R., Akarte, M.M., Raut, D.N. (2015). Framework to evaluate manufacturing capability using analytical hierarchy process. The International Journal of Advanced Manufacturing Technology, 76(1-4), 565-576.

20. Wang, J.J., Miao, Z.H., Cui, F.B., Liu, H.C. (2018). Robot Evaluation and Selection with Entropy-Based Combination Weighting and Cloud TODIM Approach. Entropy 2018, 20(5), 349-366.

21. Garetti, M., Taisch, M. (2012). Sustainable manufacturing: trends and research challenges. Production Planning \& Control, 23(2-3), 83-104.

22. Muhammad, A., Takashi, I. (2018). Scheduling for sustainable manufacturing: A review. Journal of Cleaner Production, 205, 866-883.

23. Fisher, O., Watson, N., Porcu, L., et al. (2018). Cloud manufacturing as a sustainable process manufacturing route. Journal of Manufacturing Systems, 47, 53-68.

24. Wang, L., Mohammed, A., Wang, X. V., et al. (2018). Energy-efficient robot applications towards sustainable manufacturing. International Journal of Computer Integrated Manufacturing, 31(8), 692-700.

25. Xu, W., Liu, Q., et al. (2017). Energy Condition Perception and Big Data Analysis for Industrial Cloud Robotics. Procedia CIRP, 61, 370-375.

26. Luck, M.M. (2012, June). Efficient Correlation-Aware Service Selection. ICWS 2012: IEEE International Conference on Web Services, USA, Hawaii.

27. Liu, J., Chen, Y., et al. (2018). An approach for service composition optimisation considering service correlation via a parallel max-min ant system based on the case library. International Journal of Computer Integrated Manufacturing, 31(12). 1174-1188.

28. Li, D., Ye, D., Gao, N., Wang, S. (2019). Service selection with qos correlations in distributed servicebased systems. IEEE Access, 7, 88718-88732.

29. Huang, D. (2019). A Supplier Selection Method Based on AHP. Journal of Physics: Conference Series, 1176, 042055 .

30. Chen, L., Yang, C., Huang, W., et al. (2016, December). Selecting Training method of a rehabilitation robot Based on fuzzy comprehensive evaluation. Proceedings of the 5th International Conference on Mechanical Engineering, Materials and Energy, China, Hong Kong.

31. Li, Y., Dong, S. (2015, August). Study on Supplier Selection of Manufacturing in Lean Closed-Loop Supply Chain. Proceedings of the Ninth International Conference on Management Science and Engineering Management, Germany, Berlin.

32. Rashid, T., Beg, I., Husnine, S. M. (2014). Robot selection by using generalized interval-valued fuzzy numbers with TOPSIS. Applied Soft Computing, 21, 462-468.

33. Cheng, H., Xu, W., Ai, Q., et al. (2017). Manufacturing Capability Assessment for HumanRobot Collaborative Disassembly Based on MultiData Fusion. Procedia Manufacturing, 10, 26-36.

34. Sakthivel, G., et al. (2018). Application of failure mode and effect analysis in manufacturing industry An integrated approach with FAHP-fuzzy TOPSIS and FAHP-fuzzy VIKOR. International Journal of Productivity and Quality Management, 24(3), 398 423.

35. Wang, S., Zhang, X., Xu, W., Liu, A., Zhou, Z., Pham D. (2018, March). Energy-efficient concurrent assessment of industrial robot operation based on association rules in manufacturing. 2018 IEEE 15th International Conference on Networking, Sensing and Control (ICNSC), China, Zhuhai.

36. Xie, L., Jiang, X., Xu, W., Wei, Q., Li, R., Zhou, Z. (2015). Dynamic Assessment of Sustainable Manufacturing Capability for CNC Machining Systems in Cloud Manufacturing. Advances in Production Management Systems: Innovative Production Management Towards Sustainable Growth, 460, 396-403.

37. Zhang, Y., Zhao, T., Zhang, Z., et al. (2017). Modeling and dynamic assessment on sustainable development of drainage enterprise: Application of a coupled system dynamics- comprehensive assessment model. Journal of Cleaner Production, 141, 157-167.

38. LI, J., Meng, X., Yang, D. (2015). An SSUT Network Security Situation Awareness Method Based on Supporting Extended Index System. Microelectronics \& Computer, 2, 15-19.

39. Wang, R., Jiang, N. (2018). An Improved Quantitative Method of Military Training Effect Qualitative Evaluation Indexes. Command Control and Simulation, 274 (4), 62-66.

40. Reshef, D.N., Reshef, Y.A., Finucane, H.K. (2011). Detecting Novel Associations in Large Data Sets. Science, 334 (6062), 1518-1524.

41. Shao, F.B., Li, K.P. (2016). A Complex Network Model for Analyzing Railway Accidents Based on the Maximal Information Coefficient. Communications in Theoretical Physics, 66(4), 459-466.

42. Guo, Y.J., Tang, H.Y., Qu, D.G. (2010). Dynamic comprehensive evaluation method and its application based on minimal variability. Systems Engineering \& Electronics, 32(6), 1225-1228.

43. Pham, D.T., Castellani, M. (2009). The bees algorithm: Modelling foraging behaviour to solve continuous optimization problems. Proceedings of the Institution of Mechanical Engineers, Part C: Journal of Mechanical Engineering Science, 223(12), 29192938.

44. Xu, W., Tian, S., Liu, Q., et al. (2016). An improved discrete bees algorithm for correlation-aware service aggregation optimization in cloud manufacturing. The 
International Journal of Advanced Manufacturing

Technology, 84(1-4). 17-28.

45. Saaty, T.L. (2004). Fundamentals of the analytic network process-multiple networks with benefits. Journal of Systems Science and Systems Engineering, 13(3), 348-379. 


\section{Figures}

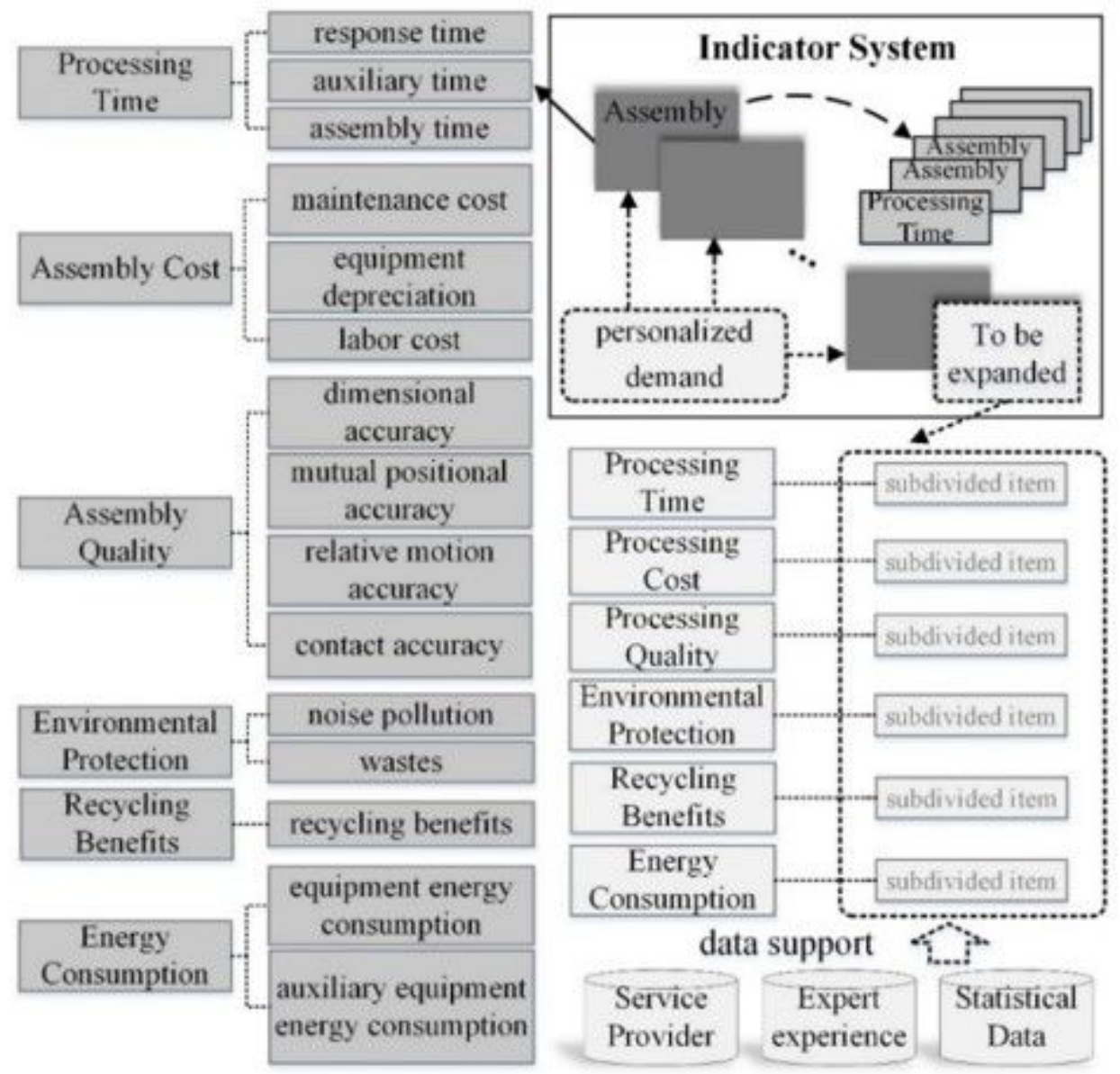

\section{Figure 1}

Extensible Multi-Dimensional Indicators System 


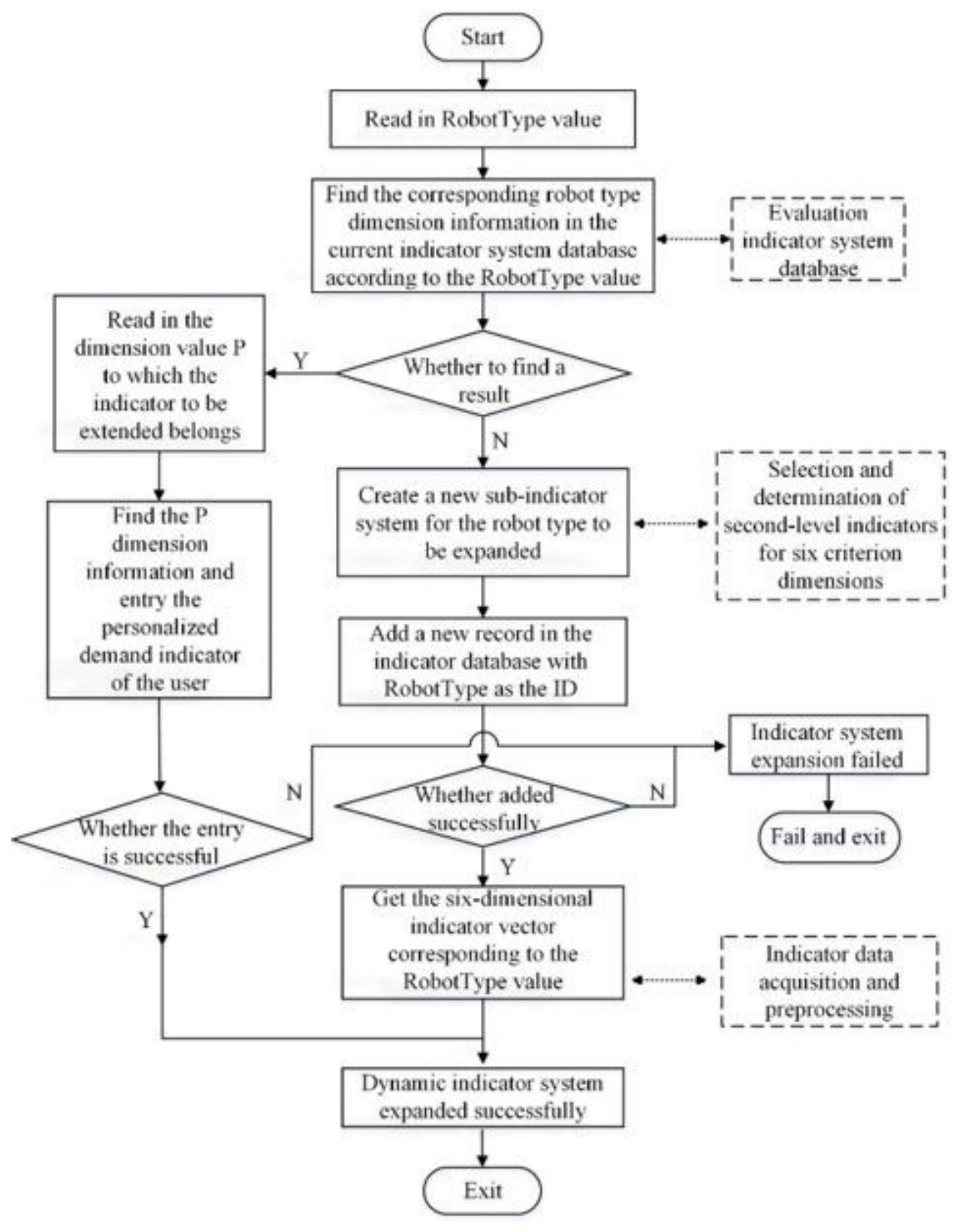

Figure 2

Extension Mechanism of Dynamic Indicator System 


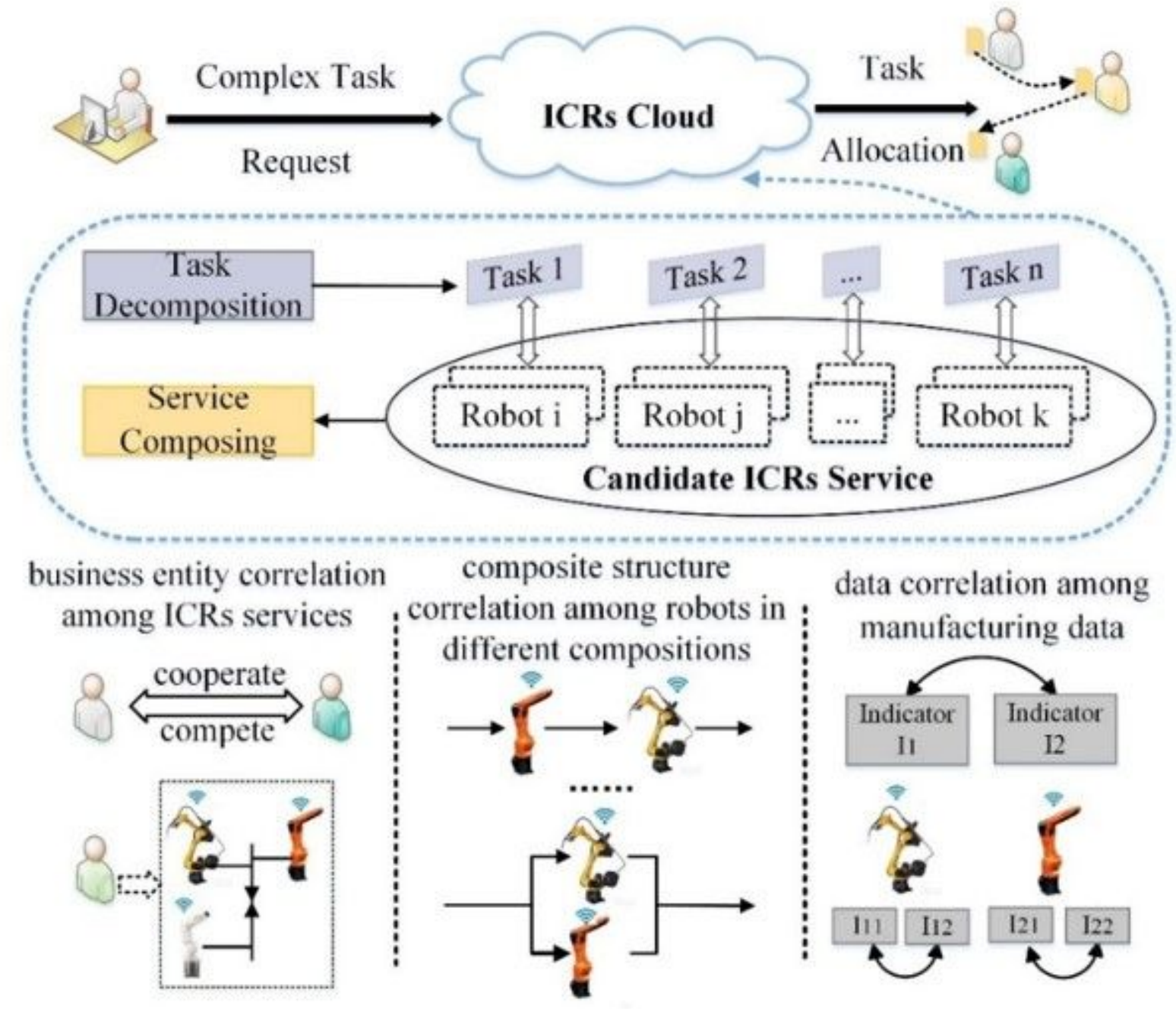

Figure 3

Manufacturing Capability Correlation Relationships in ICRs Composition 


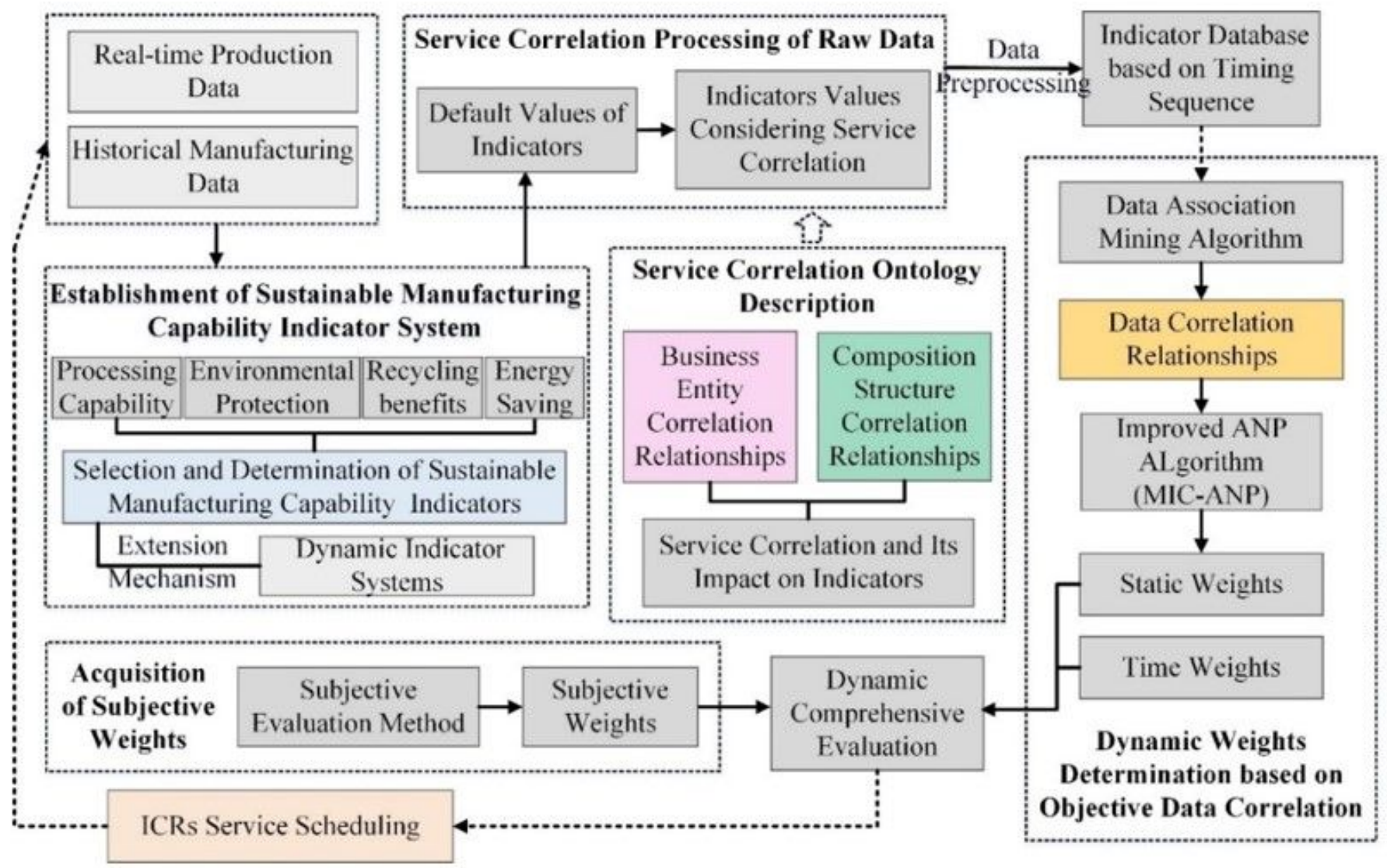

Figure 4

Correlation Assessment Model 


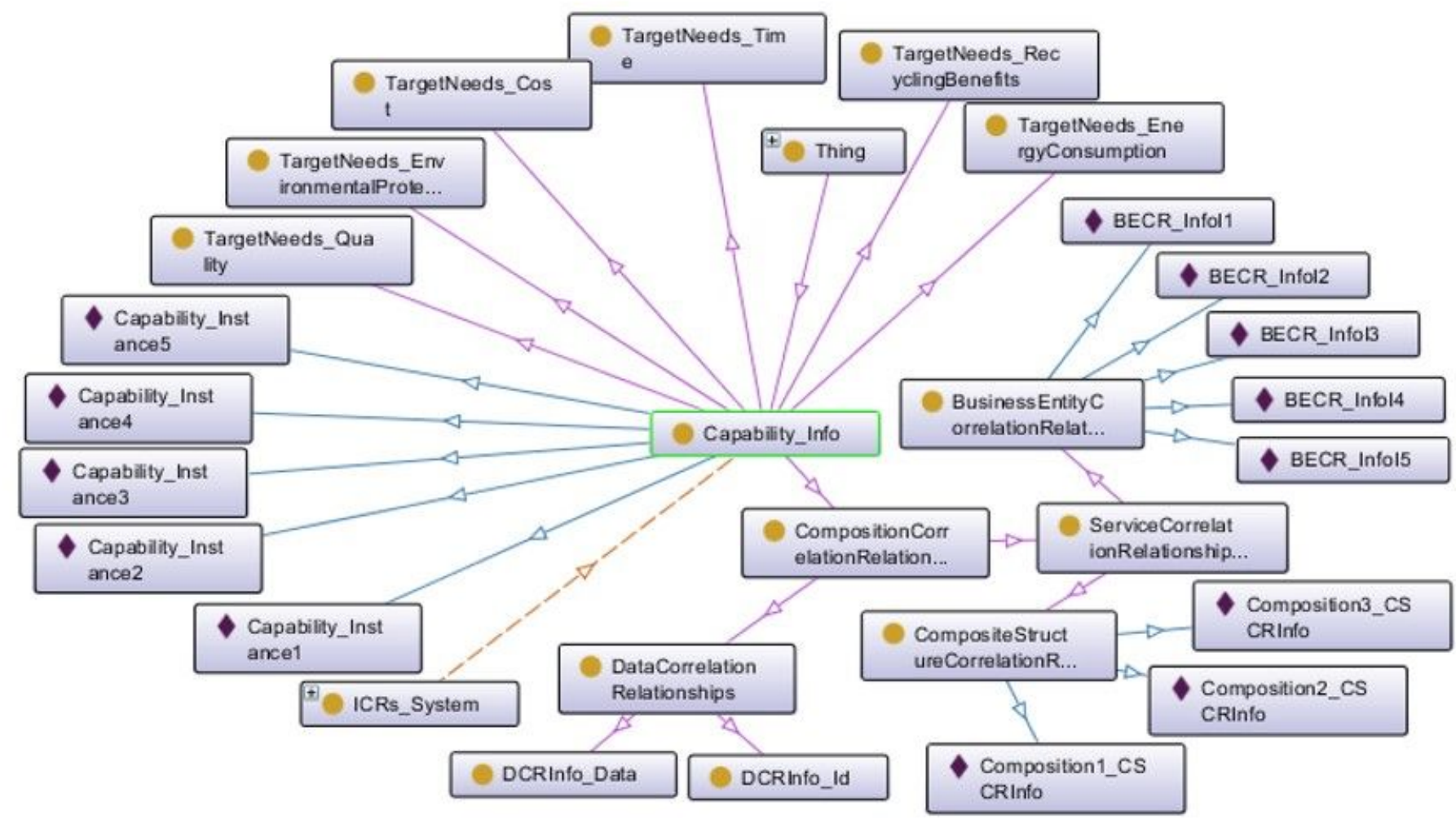

Figure 5

The Structure of Manufacturing Capability Information Ontology

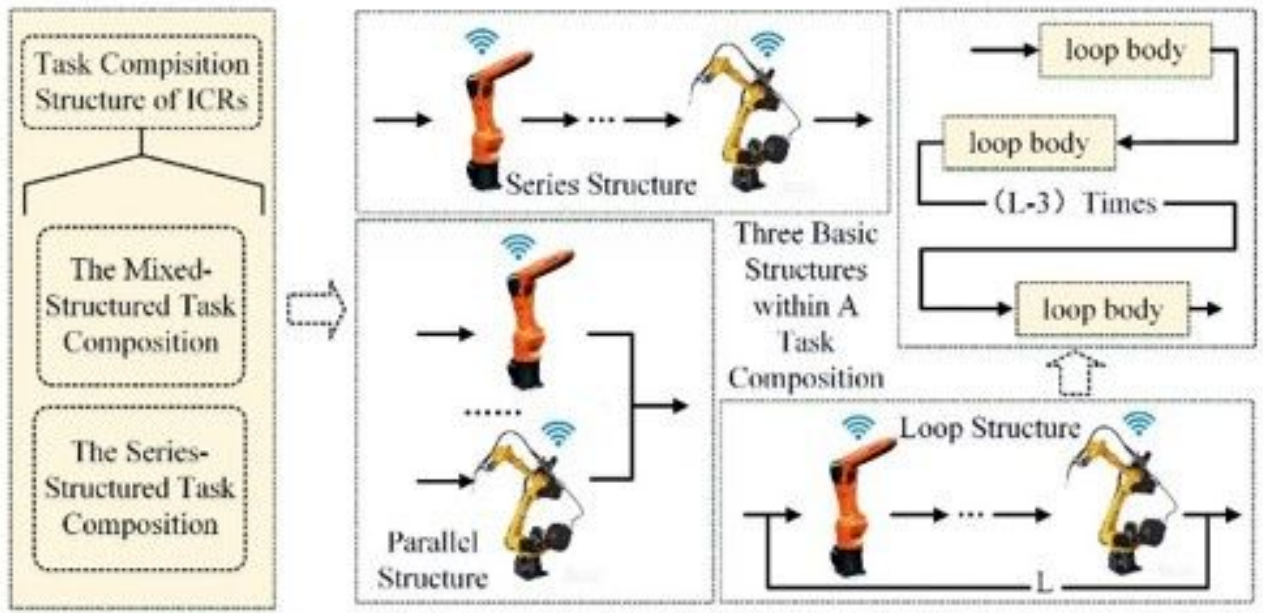

Figure 6

The Overall Structure of ICRs Composition 
Algorithm function: Solve $\theta$ Under Constraints and Reduce Data Correlation Mining Results

Input: Mining results of ICRs Sustainable Manufacturing Capability Data Correlation

$M I C=\left\{M I C\left(x_{i}, x_{j}\right)\right\}$, Parameter Setting at The End of Elimination Ratio $u=0.2$.

Output: Data Correlation Reduction Results $M I C^{\theta}=\left\{M \Pi C\left(x_{i}, x_{j}\right)\right\}$.

1. $M=\{m\} \leftarrow M I C=\left\{M \Pi C\left(x_{i}, x_{j}\right)\right\}$, sort $M$ in descending order to get its maximum and minimum values $M^{m a x}$ 与 $M^{\min }$.

2. Calculate the current $\theta$ value, $\theta=\left(M^{m a x}-M^{\min }\right) * u$.

3. Get the distribution of $M$ on the numerical intervals $[0, \theta)$ and $[\theta, 1], n_{0, \theta}(m), n_{\theta, 1}(m)$.

4. If $\frac{n_{\theta, 1}(\text { mic })}{n_{0, \theta}(m i c)} \geq 1, \quad \theta^{*}=\theta$.

5. Else $M=\{m\} \leftarrow M=\{m, \quad m<\theta\}$, back to Step 1 .

6. Output $M I C^{\theta}=\left\{M I C\left(x_{i}, x_{j}\right)\right\}, M I C\left(x_{i}, x_{j}\right) \geq \theta^{*}$, End algorithm.

Figure 7

The Pseudo-code for Solving $\theta$ and Reducing Data Correlation Results

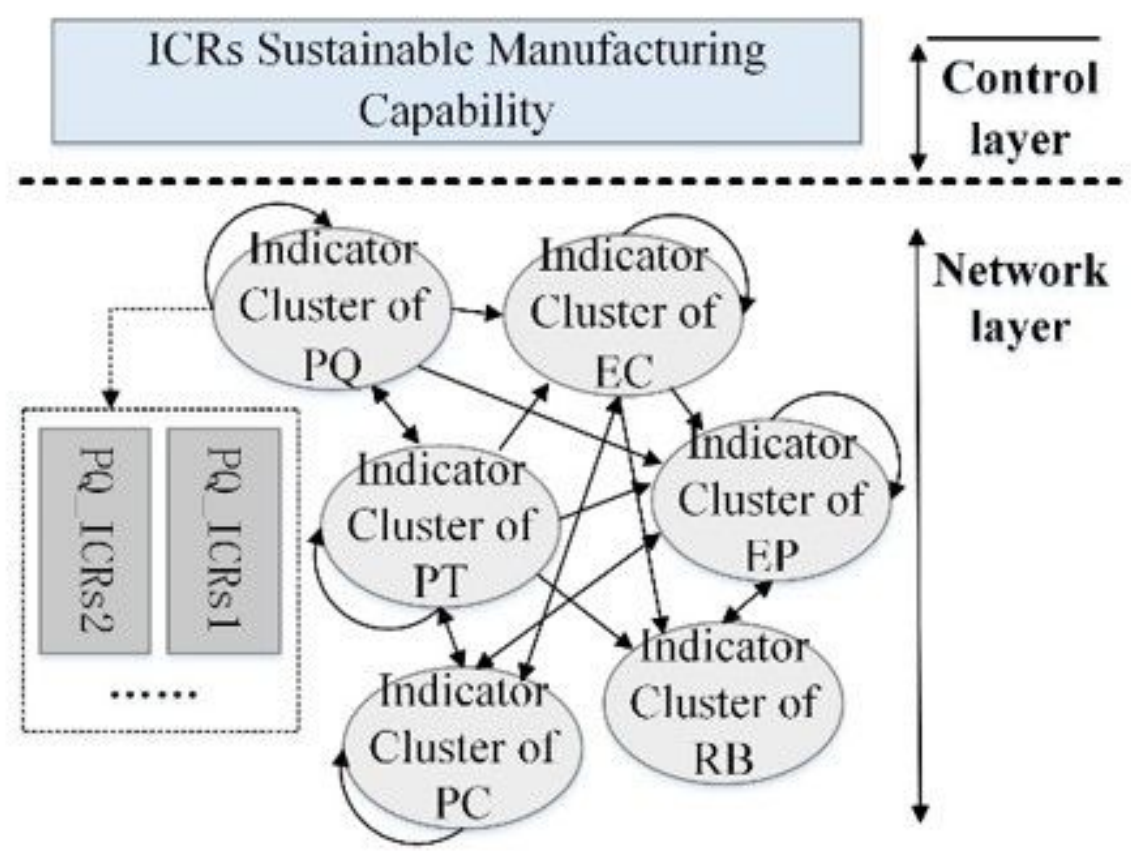

Figure 8 
The Network Structure of Indicators

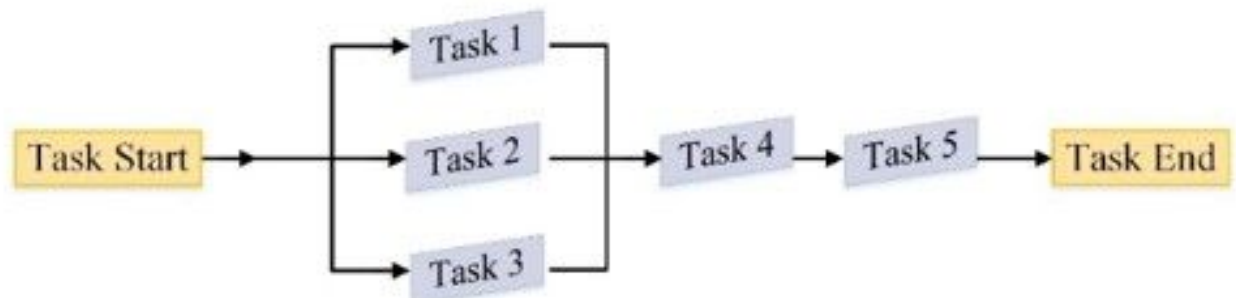

\section{Figure 9}

Assembly Task Decomposition Planning

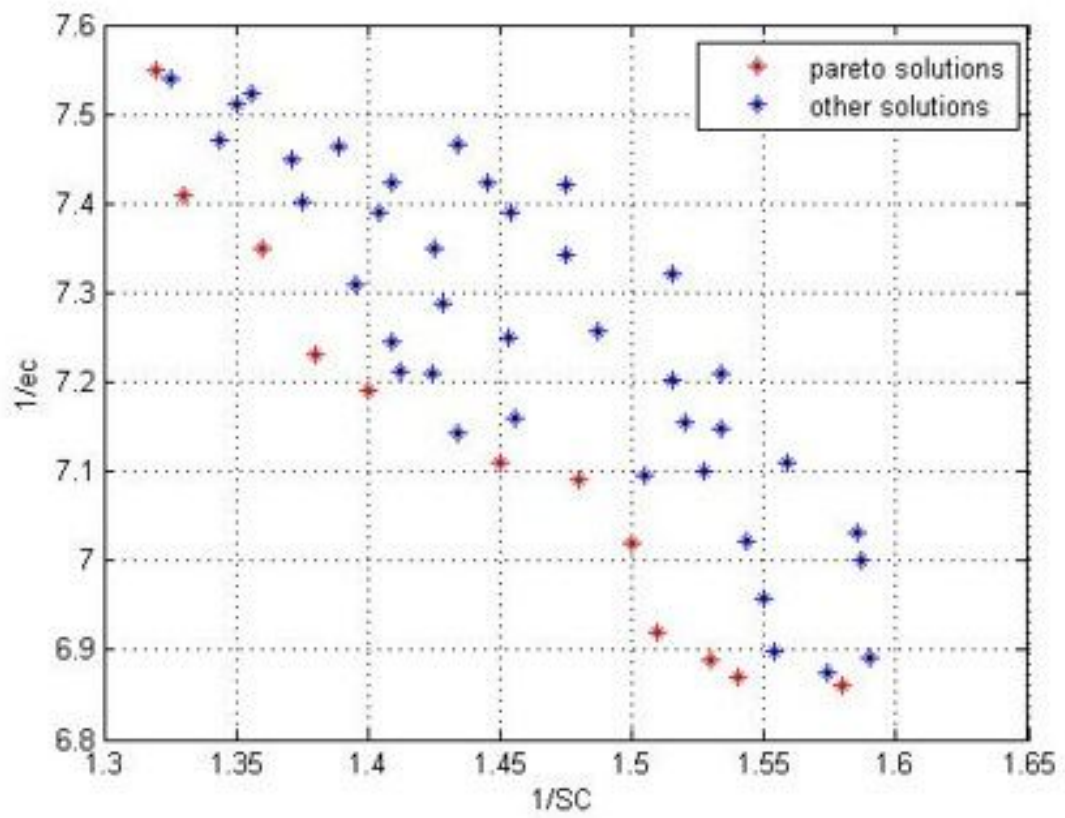

Figure 10

The Solution Space under Enumeration Method 


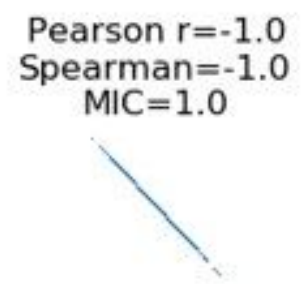

Pearson $\mathrm{r}=0.0$

Spearman $=0.0$ $\mathrm{MIC}=0.4$

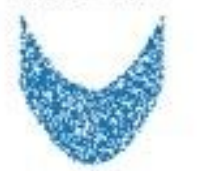

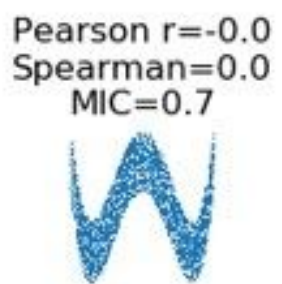

Pearson $r=-0.0$

Spearman $=-0.0$

$\mathrm{MIC}=0.4$

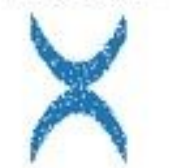

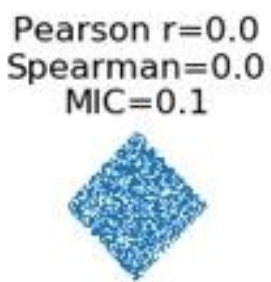

Pearson $r=0.0$ Spearman $=0.0$ $\mathrm{MIC}=0.6$

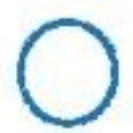

\section{Figure 11}

The Results of Three Data Correlation Mining Methods

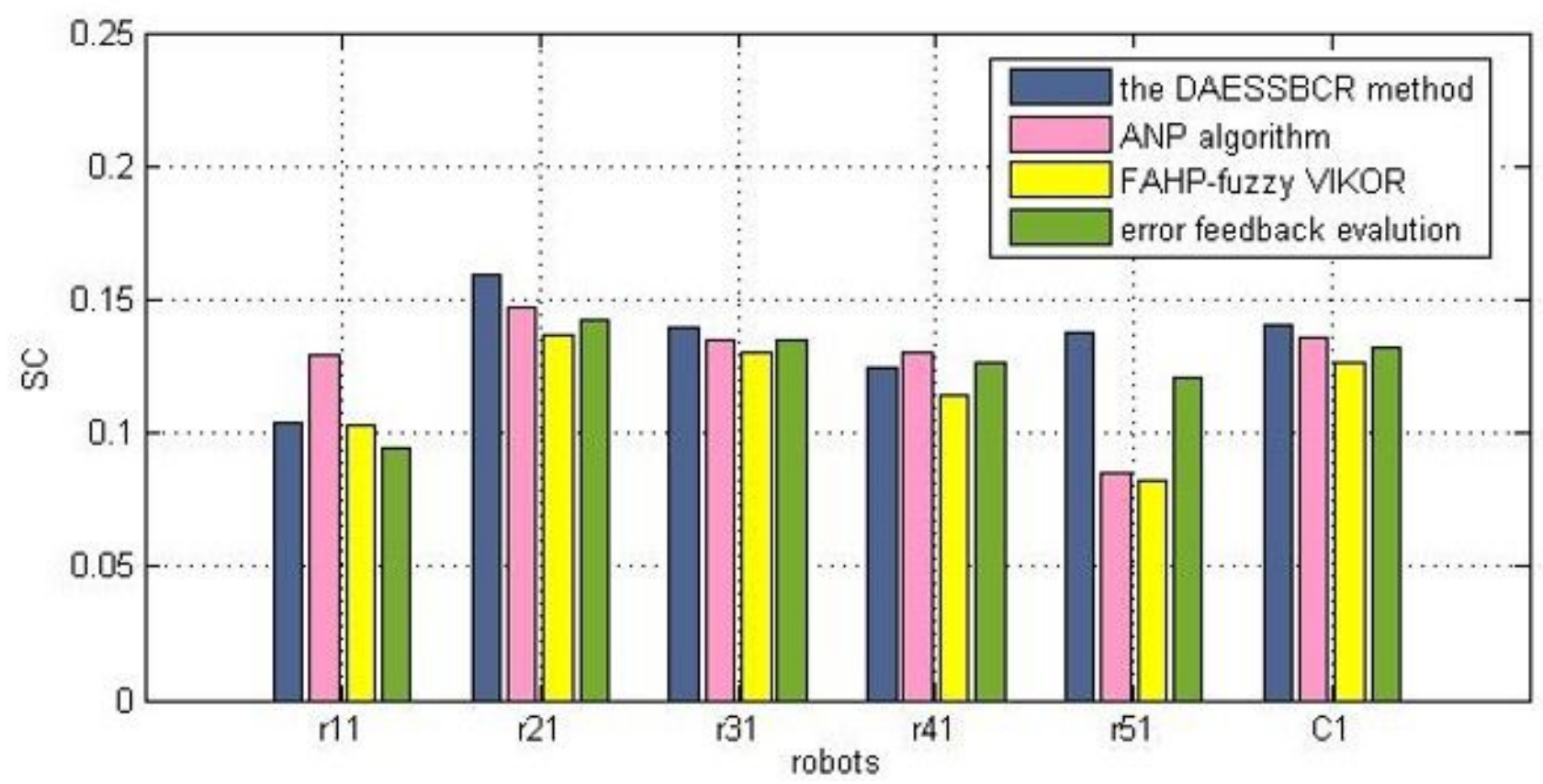

Figure 12

The Sustainable Manufacturing Capability under Different Evaluation Methods 


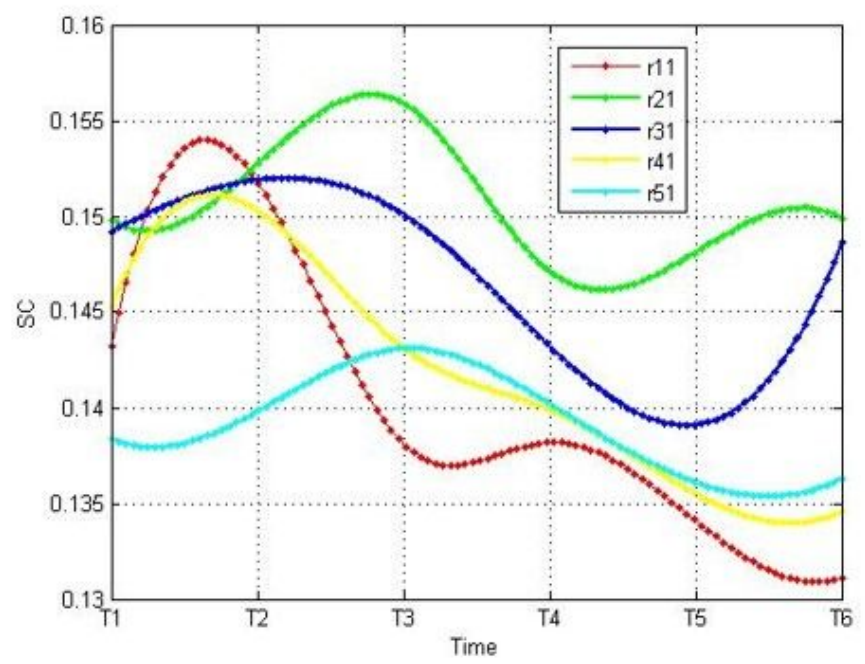

(a) The DAESSBCR Method

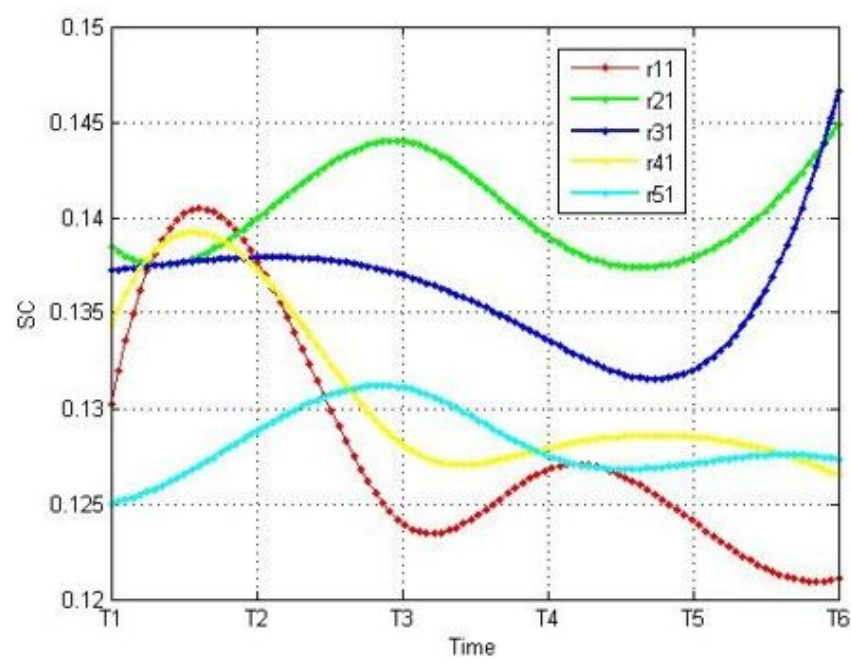

(b) Error Feedback Evaluation

\section{Figure 13}

The Sustainable Manufacturing Capability Change Curve under Different Methods 


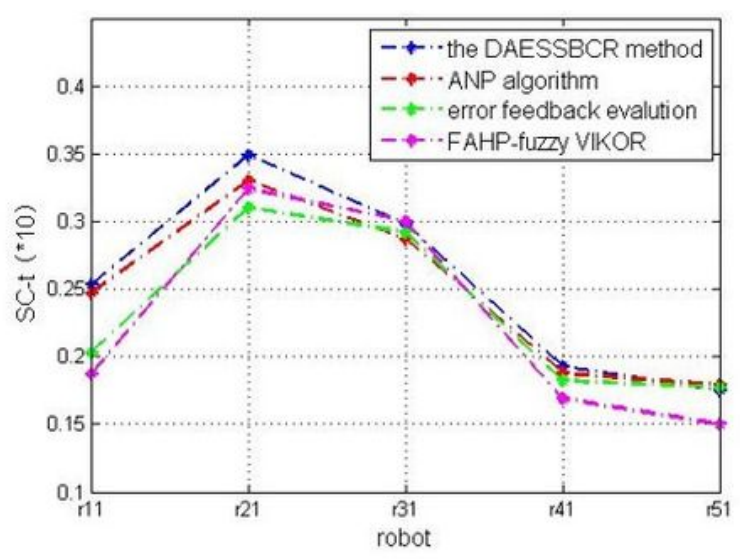

(a) Processing Time Capability

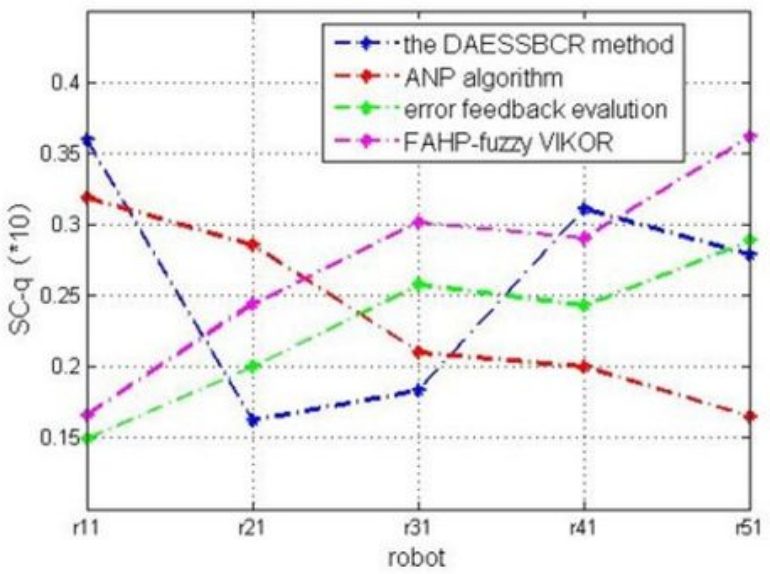

(c) Processing

Quality Capability

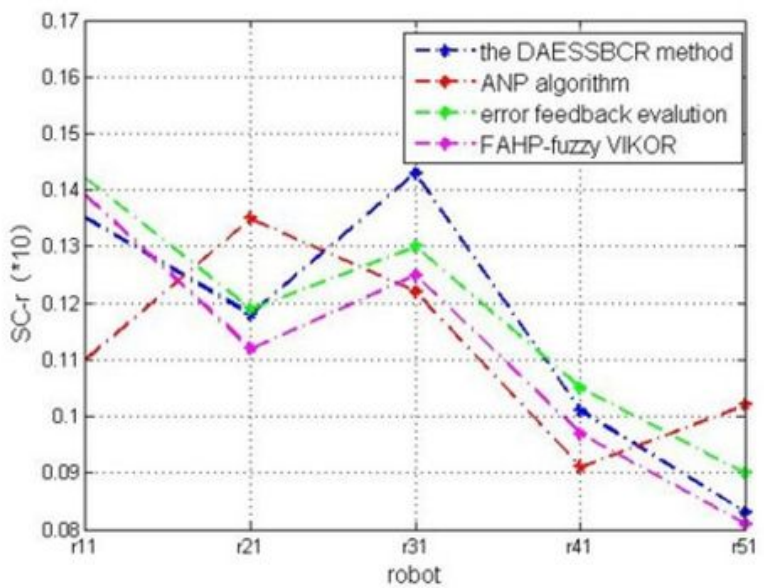

(e) Recycling Capability

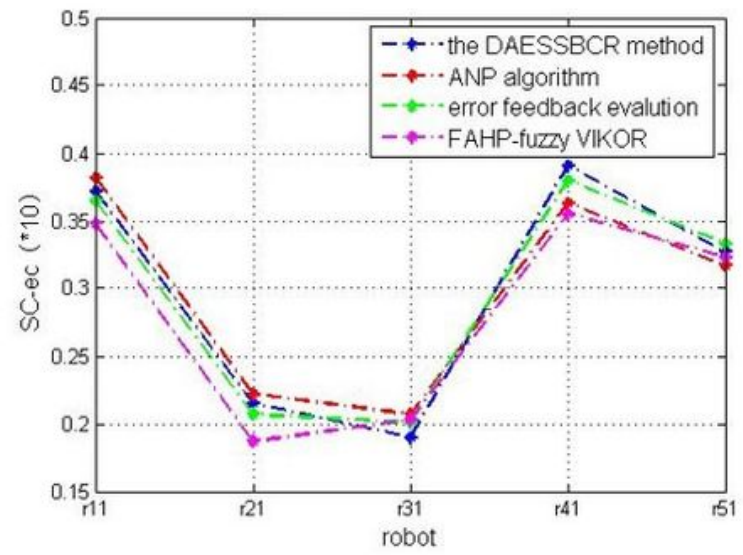

(b) Energy Consumption Capability

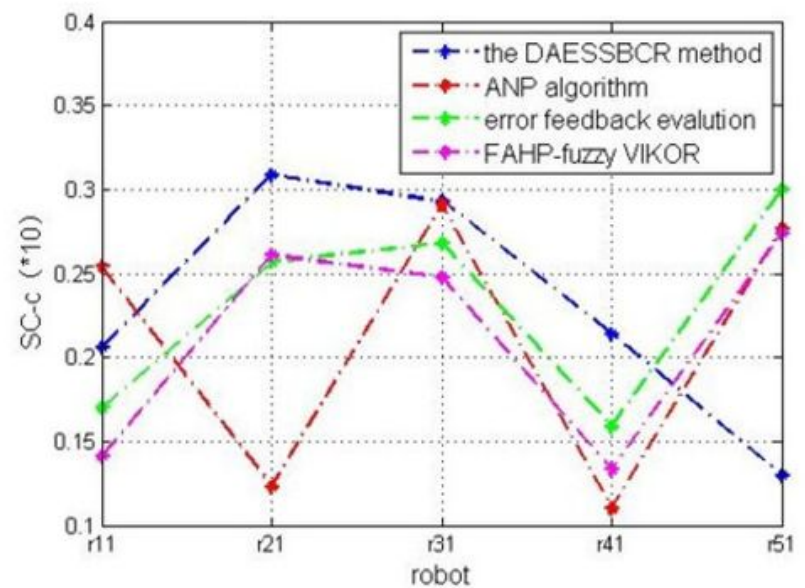

(d) Processing Cost Capability

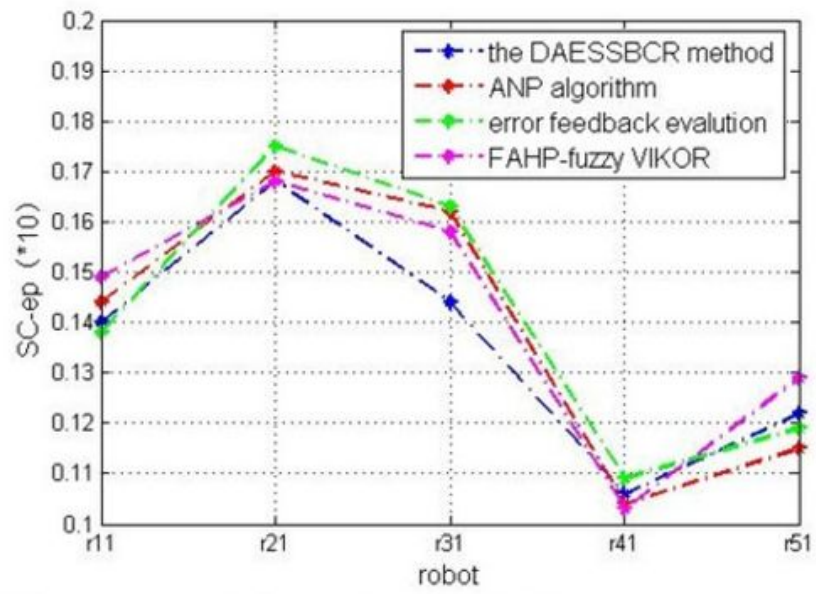

(f) Environmental Protection Capability

\section{Figure 14}

The Evaluation Results of Four Methods in Different Capability Aspects 


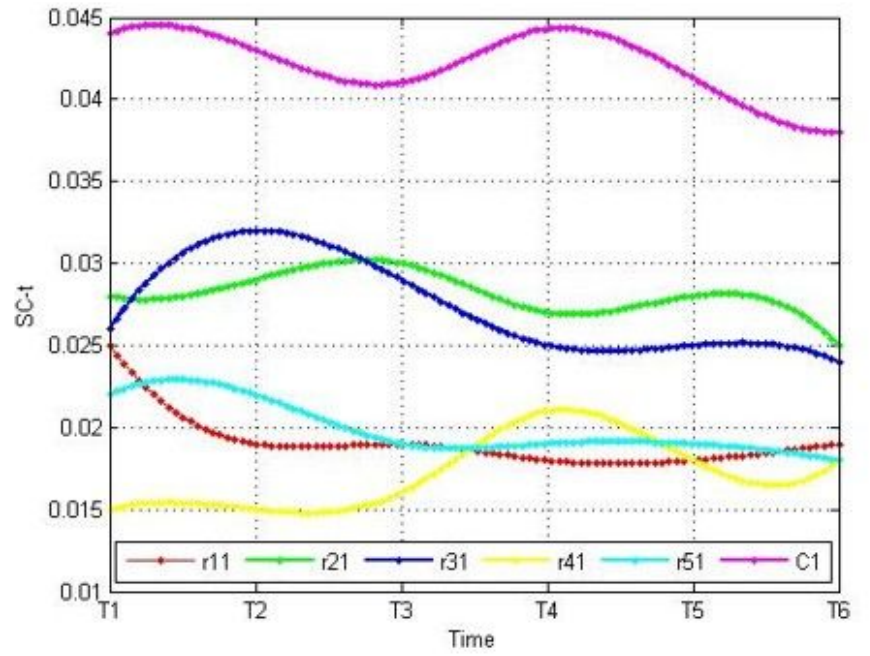

(a) The DAESSBCR Method

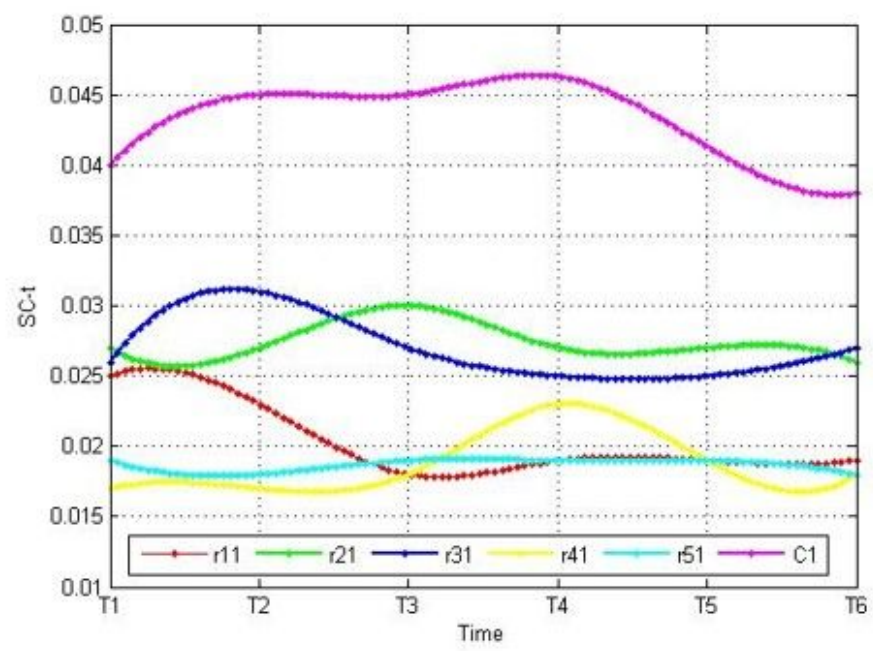

(b) Error Feedback Evaluation

\section{Figure 15}

The Processing Time Capability Change Curve under Different Methods

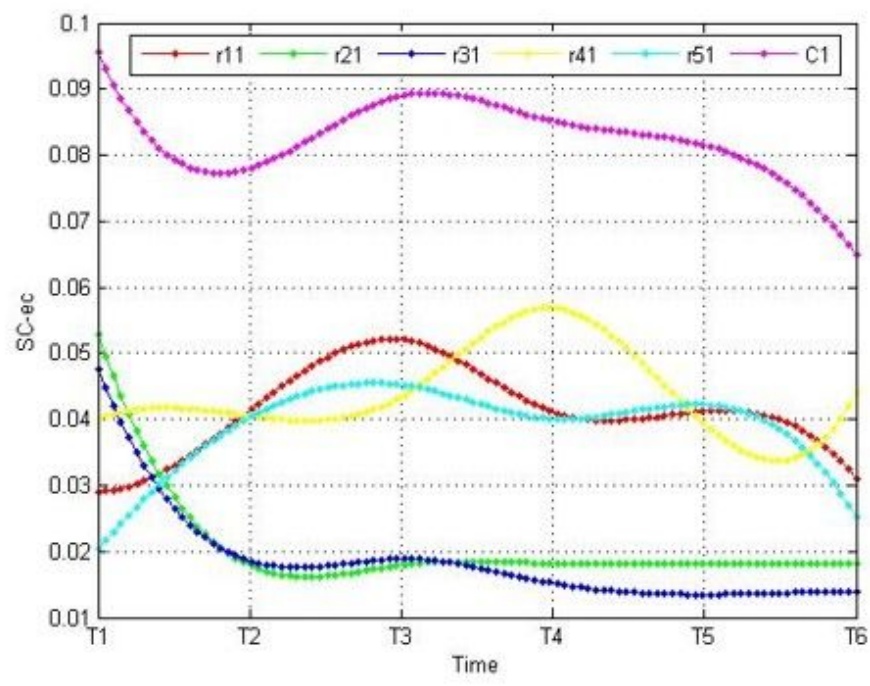

(a) The DAESSBCR Method

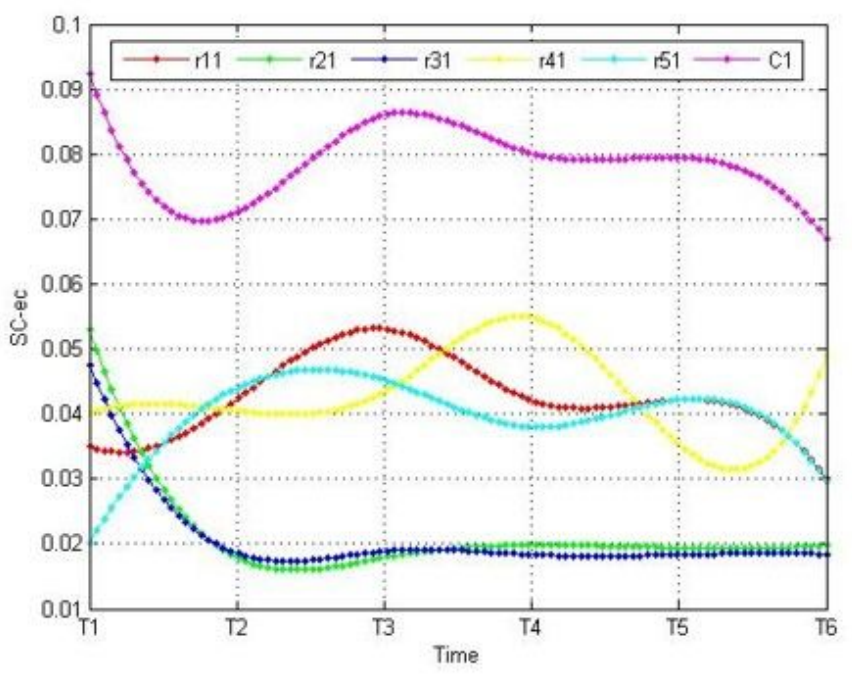

(b) Error Feedback Evaluation

Figure 16

The Energy Saving Capability Change Curve under Different Methods 


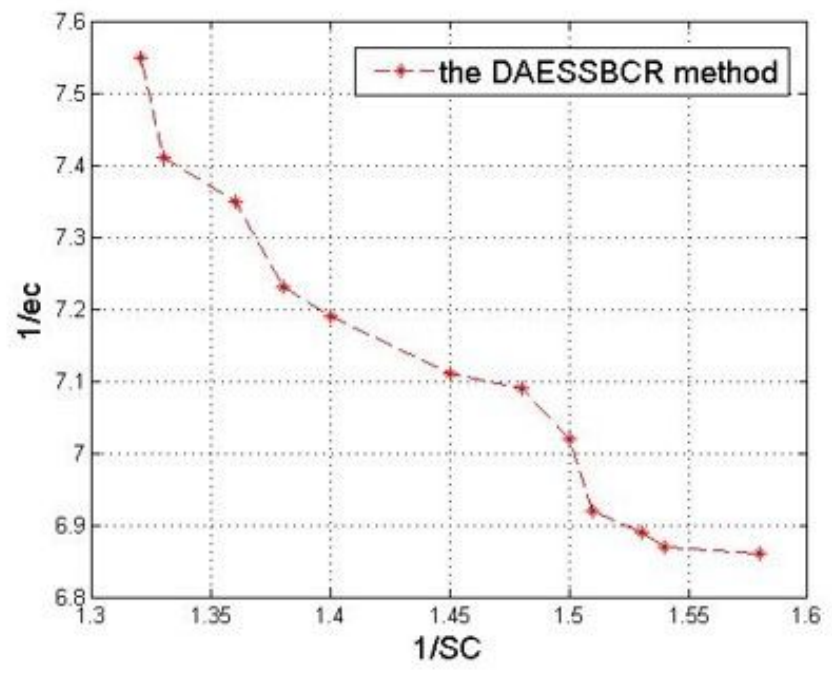

(a) The DAESSBCR Method

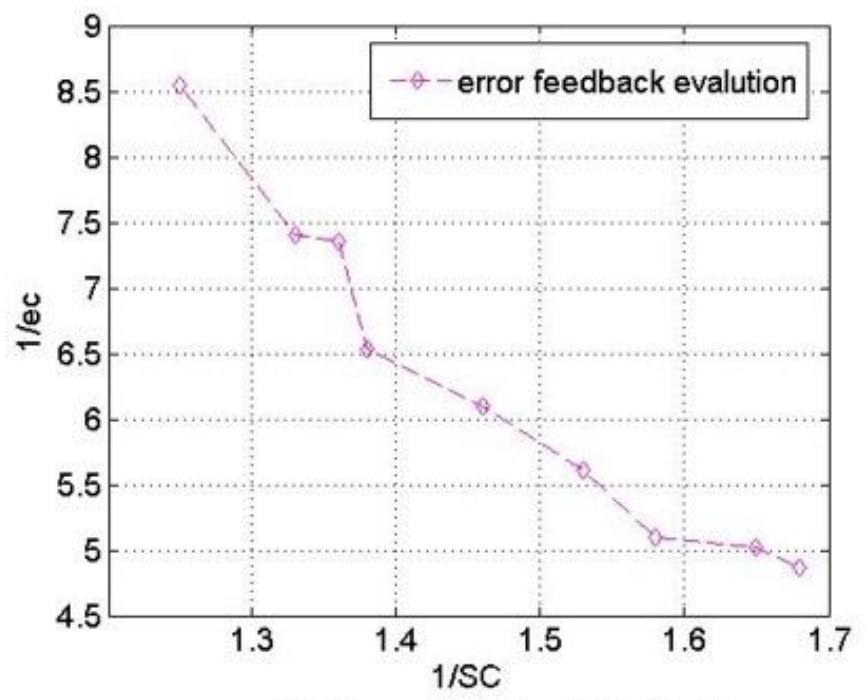

(c) Error Feedback Evaluation

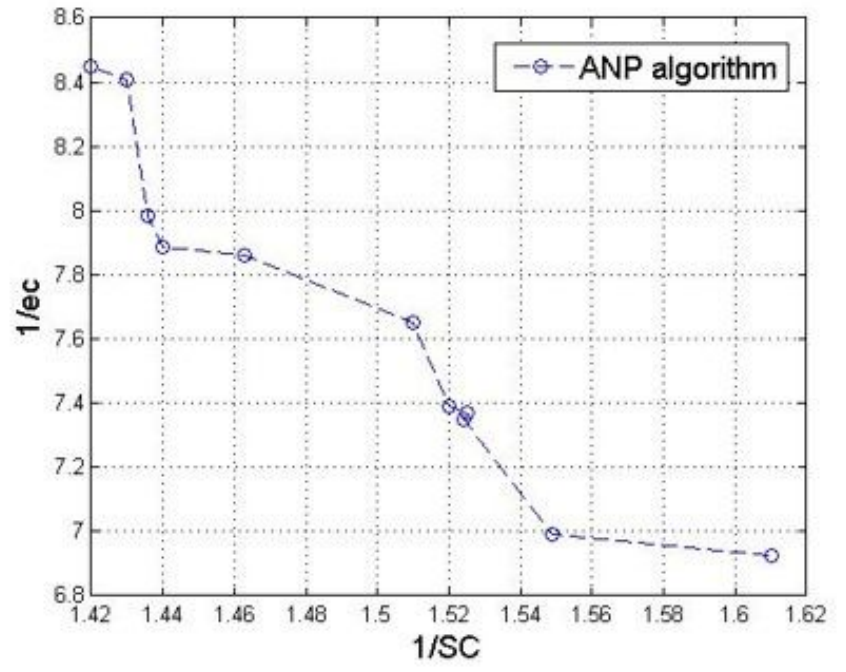

(b) ANP Algorithm

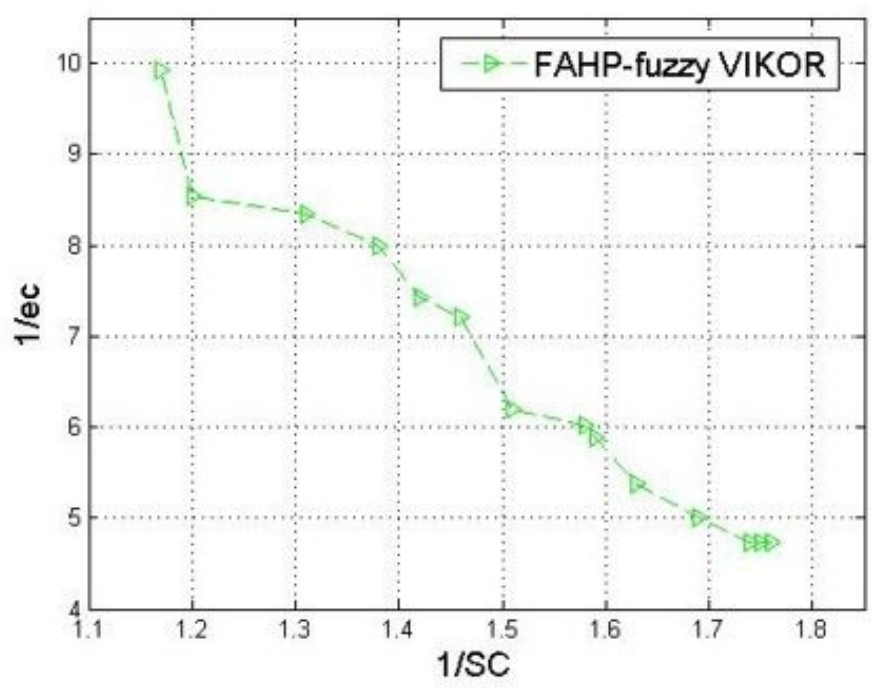

(d) FAHP-fuzzy VIKOR

Figure 17

The Pareto Fronts obtained based on Different Evaluation Methods 